\title{
Propagation and Reconstruction of Reentry Uncertainties Using Continuity Equation and Simplicial Interpolation
}

\author{
Mirko Trisolini* and Camilla Colomboì \\ Polytechnic University of Milan, Milan, 20133, Italy
}

https://doi.org/10.2514/1.G005228

\begin{abstract}
This Paper proposes a continuum-based approach for the propagation of uncertainties in the initial conditions and parameters for the analysis and prediction of spacecraft reentries. Using the continuity equation together with the reentry dynamics, the joint probability distribution of the uncertainties is propagated in time for specific sampled points. At each time instant, the joint probability distribution function is then reconstructed from the scattered data using a gradient-enhanced linear interpolation based on a simplicial representation of the state space. Uncertainties in the initial conditions at reentry and in the ballistic coefficient for three representative test cases are considered: a three-state and a six-state steep Earth reentry and a six-state unguided lifting entry at Mars. The Paper shows the comparison of the proposed method with Monte Carlo-based techniques in terms of quality of the obtained marginal distributions and runtime as a function of the number of samples used.
\end{abstract}

\section{Introduction}

$\mathbf{T}$ HE study and prediction of reentry trajectories is a challenging and complex task. Several factors may influence the accuracy of these predictions, such as the knowledge of the initial entry conditions, the ballistic coefficient of the satellite, and the density of the atmosphere. The uncertainties associated with these parameters can affect the evolution of the reentry trajectory and influence the prediction of several quantities of interest such as the impact location and the mechanical and thermal loads on the spacecraft. It is thus important to include statistical verification inside the mission design process, considering the effects of uncertainties in assessing possible off-nominal scenarios. Combining this analysis with operational constraints and safety margins can ultimately improve the safety and robustness of mission designs. This type of statistical analysis can be applied to several aspects of the reentry problem. The design of crewed vehicles and their navigation algorithms can strongly benefit from an uncertainty analysis given the strict requirements on the sustainable loads and on the landing location [1-5]. The design of exploration probes and sample return missions can also gain from uncertainty quantification to improve the robustness of the mission [6-8], with the aim to increase the landing mass and precision, and to provide more robust designs for the thermal protection and parachute deployment systems. Finally, even the destructive reentry of satellites and the prediction of their reentry footprint, in particular for uncontrolled reentries, can benefit from uncertainty assessment. Until 2013, there had been an average of 93 uncontrolled reentries per year [9], and with the increasing space activities of the past few years, the frequency of reentering objects is destined to grow rapidly. Despite these objects usually pose a marginal risk for people on the ground, it is still necessary to verify their compliance with the casualty risk regulations [10]. This ultimately means studying the breakup of the spacecraft and the demise of its parts [11-13]. Considering the effects of uncertainties, it is possible to assess the demise probability of specific components [14] and the statistical distribution of the casualty area of surviving fragments.

Presented as Paper 19-0409 at the 29th AAS/AIAA Space Flight Mechanics Meeting, Ka'anapali, HI, January 13-17, 2019; received 3 March 2020; revision received 22 October 2020; accepted for publication 17 November 2020; published online Open Access 20 January 2021. Copyright (C 2020 by the authors. Published by the American Institute of Aeronautics and Astronautics, Inc., with permission. All requests for copying and permission to reprint should be submitted to CCC at www.copyright.com; employ the eISSN 1533-3884 to initiate your request. See also AIAA Rights and Permissions www.aiaa.org/randp

*Postdoc Research Fellow, Department of Aerospace Science and Technology, via La Masa 34.

${ }^{\dagger}$ Associate Professor, Department of Aerospace Science and Technology, via La Masa 34.
The traditional procedure to assess uncertainties is based on a Monte Carlo (MC) dispersion analysis, where, through a large number of simulations over randomly sampled initial conditions and parameters, the joint probability density function (PDF) is estimated through a frequentist approach. This types of simulations provide a reliable way to estimate the evolution of uncertainties given their straightforward implementation, and they can effectively capture nonlinearities in the system. However, in general, to obtain convergent statistics, the number of MC simulations must increase. Consequently, for multidimensional state spaces and nonlinear dynamics, such as the ones associated with reentry scenarios, they can become expensive $[15,16]$. Nonetheless, many space missions' uncertainty analyses have been carried out using a Monte Carlo approach. Examples are the mission design of the Mars Pathfinder [6], of the Mars Science Laboratory [7] missions, and of the future Mars2020 exploration mission [8]. Simulation software such as the NASA Dynamics Simulator for Entry, Descent, and Surface Landing (DESENDS) [17] uses a Monte Carlo-based dispersion analysis. In addition, MC simulations have been extensively used for the design verification of entry guidance algorithms $[2,3,18-20]$. In these works, the performance, robustness, and reliability of the algorithm is tested considering a dispersion in the initial conditions and parameters of the reentry. The results of the MC simulations in terms of mean and standard deviation are compared to the target for the landing accuracy, and the number of occurrences for violation of specified safety thresholds is considered. Also, destructive reentry codes such as ESA DRAMA [13] perform reentry analyses using a MC approach for the design and verification of satellite compliance to the casualty risk regulations.

Instead of MC simulation, several other techniques have been applied to the propagation of uncertainties [16], for example, unscented transformation (UT), where only a few, deterministically chosen, samples are propagated (the sigma points). From these samples, it is then possible to obtain a second-order approximation of the first two moments of the probability distribution. Other methodologies are instead based on the creation of a surrogate model that can more efficiently estimate the uncertainty when compared to MC [2123]. An example of such methodologies is polynomial chaos expansion [24,25], where the inputs and outputs of a system are represented via series approximation. They provide an efficient way to propagate uncertainties building an explicit functional representation of the output uncertainty with respect to the inputs. These techniques are computationally efficient, even in high dimensions, even though they rely on the estimation of the uncertainties through a frequentist approach as MC does. Another common uncertainty propagation technique is based on differential algebra (DA) [26], which allows the computation of arbitrary order expansion with respect to the initial condition. DA can be used in conjunction with MC simulations, substituting them with Taylor expansion. Gaussian mixture models [16] can also be used to propagate uncertainties. In this method, the 
nonlinearity and non-Gaussian behavior of the probability density function is captured by using multiple Gaussian distributions. However, the effects of nonlinearities have to be considered by possibly splitting and merging the different components of the mixture.

The approach we propose uses the continuity equation to directly propagate the probability density along with the dynamics of the system, thus obtaining a systematic evolution of the probability density. This methodology has been applied in the study of the dynamical evolution and formation of stellar systems, planetary ring structures, and interplanetary dust [27-29]. Additionally, it has been used for the propagation of space debris fragments following a breakup of satellites in orbit $[30,31]$ and for the propagation of uncertainties in planetary reentries [32-35]. Such a methodology is opposed to MC simulations where the distribution is approximated through many realizations; while with MC methods we propagate individual realization of the initial PDF, with a continuum-based approach, we propagate the ensemble of realizations. When not only the first moments of the distribution but also its whole shape is of interest are needed, MC simulations require a large number of samples. Instead, the proposed continuumbased propagation method allows the knowledge of the probability density at specific points in the state space to enable a reconstruction of the probability density function with a reduced number of samples.

The challenge of using a method based on the continuity equation is the postprocessing of the data. As we are propagating a finite set of initial points, with their probability density, it is then necessary to reconstruct this density in the state space starting from discrete values. In [32], a reconstruction methodology has been proposed, which replicates the binning process of MC simulations. For each bin, the density is computed as the mean of the density values of the data points contained in it. This method can be used if the PDF data are uniformly distributed in each bin in all dimensions and if the enclosed volume of the scattered data in each bin is equal [36]. However, even if the initial distribution of samples is uniform, its evolution in time does not necessarily remain uniform, and the enclosed volume of the data in each bin does not remain equal. Therefore, such a methodology generates results which present poor agreement with corresponding Monte Carlo simulations $[32,36]$. In addition, such a methodology still uses a number of samples comparable to Monte Carlo methods. We instead propose reconstructing the density using a simplex-based linear interpolation methodology [37] that uses the concept of $\alpha$ shape [38], which adapts to the evolution of the shape of the state space volume. In addition, we increase the accuracy of the linear interpolation by including the derivative information into the linear interpolation scheme using the reduced-order dual Taylor expansion [39].

The Paper presents three relevant test cases, which include uncertainties in the initial conditions, in the ballistic coefficient of the satellite, and in the atmospheric density. The first test case considers a strategic reentry on Earth using a three-state dynamics, the second test case expands the first one to a more complex dynamics, and the third test case features the lifting reentry of a probe in Martian atmosphere. The results are presented as one-dimensional and two-dimensional marginal distributions of the relevant parameters. The distribution for derived quantities of interest, such as the heat rate and the dynamic pressure, are also presented. The Paper is organized as follows. Section II describes the methodology for the propagation of the uncertainties using a continuum-based approach. Section III describes the methodology used for the reconstruction of the probability density and for the computation of the marginal probabilities. Section IV presents the application of the density propagation and reconstruction procedure to relevant test cases. Section $\underline{\mathrm{V}}$ contains the discussion and the conclusions.

\section{Uncertainty Propagation}

The proposed methodology uses the continuity equation to propagate the initial joint probability distribution function related to the uncertainties in the initial conditions and parameters and assess its evolution throughout the reentry process under the influence of the reentry dynamics. The expression for the continuity equations is [28]

$$
\frac{\partial n(\boldsymbol{x}, t)}{\partial t}+\nabla \boldsymbol{f}(\boldsymbol{x})=\dot{n}^{+}-\dot{n}^{-}
$$

where $\boldsymbol{x}$ is the vector of the state variables; $n(\boldsymbol{x}, t)$ is the joint PDF at time $t ; \boldsymbol{f}(\boldsymbol{x})$ represents the forces acting on the system and takes into account slow, continuous phenomena such as gravity and atmospheric drag; and $\dot{n}^{+}$and $\dot{n}^{-}$represent the fast and discontinuous events (i.e., sources and sinks). For the case under examination, the source and sink terms were neglected. Knowing the initial density distribution $n(\boldsymbol{x}, 0)$, Eq. (1) allows for the propagation of the density evolution in time, in a system with the equation of the dynamics. When applied to the propagation of uncertainties, the density represents the probability distribution. This is a partial differential equation (PDE) with the PDF $n(\boldsymbol{x}, t)$ being the dependent variable. Such an equation regulates the conservation of the total probability mass of the joint PDF through its spatial-temporal evolution due to the forces acting on the system. Equation (1) can be solved using the method of the characteristics (MOC), where the partial differential equation is transformed into a set of ordinary differential equations (ODEs). As it is convenient to express the evolution of the reentry trajectory using parameters such as the altitude, the relative velocity, and the flightpath angle, we follow the approach to express the continuity equation in the state space of the problem under examination, writing the divergence in rectangular coordinates [28]. In the generic case of $d$ number of variables, Eq. (1) can be rewritten in rectangular coordinates as follows

$$
\frac{\partial n}{\partial t}+\frac{\partial n}{\partial \alpha_{1}} v_{\alpha_{1}}+\cdots+\frac{\partial n}{\partial \alpha_{d}} v_{\alpha_{d}}+\left[\frac{\partial v_{\alpha_{1}}}{\partial \alpha_{1}}+\cdots+\frac{\partial v_{\alpha_{d}}}{\partial \alpha_{d}}\right] n=0
$$

where $\alpha_{i}$ are the state variables and $v_{\alpha_{i}}$ are the corresponding forces. The sink and source terms have been neglected in this case. Applying the method of characteristics, the PDE can be reduced to the following system of ODEs:

$$
\left\{\begin{array}{l}
\frac{\mathrm{d} t}{\mathrm{~d} s}=1 \\
\frac{\mathrm{d} \alpha_{1}}{\mathrm{~d} s}=v_{\alpha_{1}}\left(\alpha_{1}, \ldots, \alpha_{d}\right) \\
\vdots \\
\frac{\mathrm{d} \alpha_{d}}{\mathrm{~d} s}=v_{\alpha_{d}}\left(\alpha_{1}, \ldots, \alpha_{d}\right) \\
\frac{\mathrm{d} n}{\mathrm{~d} s}=\left[\frac{\partial v_{\alpha_{1}}}{\partial \alpha_{1}}+\cdots+\frac{\partial v_{\alpha_{d}}}{\partial \alpha_{d}}\right] n\left(\alpha_{1}, \ldots, \alpha_{d}\right)
\end{array}\right.
$$

where $s$ is the independent variable. In this Paper, the continuity equation is applied to two sets of equations of motion. First, a threestate representation models, which describes the evolution of the reentry through radius $r$, velocity $v$, and flight-path angle $\gamma$ under the influence of the planet gravity and the atmospheric drag. The dynamics of this model is described in Eq. (4) and considers a planar motion over a nonrotating planet,

$$
\left\{\begin{array}{l}
\dot{r}=v \sin \gamma \\
\dot{v}=-\frac{1}{2 \beta} \rho v^{2}-g \sin \gamma \\
\dot{\gamma}=\left(\frac{v}{r}-\frac{g}{v}\right) \cos \gamma+\frac{1}{2 \beta} \frac{C_{L}}{C_{D}} \rho v
\end{array}\right.
$$

where $\rho$ is the atmospheric density, $g$ is the gravitational acceleration, $\beta=\left(m / C_{D} S\right)$ is the ballistic coefficient, $C_{L}$ is the lift coefficient, $C_{D}$ is the drag coefficient, and $S$ is the object cross-section. Applying the MOC of Eq. (3) to this set of equations, the variation in time of the probability density $n$ is obtained as follows:

$$
\dot{n}=-\left[\frac{\partial \dot{r}}{\partial r}+\frac{\partial \dot{v}}{\partial v}+\frac{\partial \dot{\gamma}}{\partial \gamma}\right] n
$$


If we want uncertainties in additional parameters, other than reentry states, to be considered, it is necessary to include them in the equations of motion and add their contribution to the evolution of the density in Eq. (5). For example, in the case under examination, we want to consider an uncertainty over the ballistic coefficient $\beta$ and include the possibility of taking into account uncertainty in the atmospheric density (through an atmospheric correction coefficient $\xi$ ). The resulting system of equations for the propagation of the characteristics becomes as follows:

$$
\left\{\begin{array}{l}
\dot{r}=v \sin \gamma \\
\dot{v}=-\frac{1}{2 \beta} \rho(r, \xi) v^{2}-g(r) \sin \gamma \\
\dot{\gamma}=\left(\frac{v}{r}-\frac{g(r)}{v}\right) \cos \gamma+\frac{1}{2 \beta} \frac{C_{L}}{C_{D}} \rho(r, \xi) v \\
\dot{\beta}=0 \\
\dot{\xi}=0 \\
\dot{n}=-\left[\frac{v}{\beta} \rho(r, \xi)+\sin \gamma\left(\frac{v}{r}-\frac{g(r)}{v}\right)\right] n
\end{array}\right.
$$

Therefore, the result is the augmented state $(r, v, \gamma, \beta, \xi)$ to be propagated. The second model considered in this Paper is a sixstate representation, which describes the three-dimensional translational reentry over a rotating Earth. For the case under examination, we decided to express the equations of motion using the radius as the independent variable, instead of the time (de facto obtaining a five-state model). On one side, this allows showing the flexibility of the continuum propagation, and on the other, it simplifies the representation of the output of the propagation as, for example, it is more convenient to extract information at the landing instant, which corresponds to the final radius in the propagation. The set of equations of motion is the following: gravitational acceleration, respectively. The expression for the derivative of the density as a function of $r$ was not expanded for a better readability. In this expression, the primes refer to derivatives with respect to the radius. Also in this case, we have modeled the uncertainties in the ballistic coefficient and atmospheric density by including the coefficients $\beta$ and $\xi$ into the extended state of the problem.

Equations (6) and (7) must be integrated numerically, and the integration can be performed using a standard ODE solver such as Runge-Kutta. In this way, the time evolution of the density in the state space can be obtained as a function of the considered independent variable. As the solution for the reentry problem is not analytical, it is necessary to sample the uncertainty distribution in the initial states and to propagate the trajectory and the probability density for each sample point.

\section{Interpolation Method}

Once the sampled initial conditions have been propagated using Eq. (3), it is necessary to reconstruct the probability density in the domain at each time step to obtain the total uncertainty and the marginal distributions. The proposed approach is integrated with the continuum propagation described in Sec. II to reconstruct the probability density from few samples, leveraging on the knowledge of the value of the density that is propagated alongside the characteristics. As can be observed in Fig. 6 , during the evolution of the reentry trajectory, not only does the density change and deform, but also the state-space volume changes and deforms. This is why the method proposed in [32], which performs a uniform binning to estimate the probability density by averaging the value in each bin, provides inaccurate results when the state space starts to deform. In our methodology, instead, we follow the variation of the state space volume by creating a simplicial (i.e., a generalization of the notion of triangle or tetrahedron to arbitrary dimensions) representation and interpolating the scattered data. Scattered data interpolation is a complex task and is even more challenging when the considered data have more than three

$$
\left\{\begin{aligned}
\frac{\mathrm{d} \lambda}{\mathrm{d} r}= & \frac{\sin (\chi)}{r \cos (\varphi) \tan (\gamma)} \\
\frac{\mathrm{d} \varphi}{\mathrm{d} r}= & \frac{\cos (\chi)}{r \tan (\gamma)} \\
\frac{\mathrm{d} v}{\mathrm{~d} r}= & -\frac{v \rho(r, \xi)}{2 \beta \sin (\gamma)}-\frac{1}{v}\left(g_{r}(r, \varphi)+\frac{\cos (\chi) g_{\varphi}(r, \varphi)}{\tan (\gamma)}\right)+\frac{r \omega_{p}^{2} \cos (\varphi)}{v}\left(\cos (\varphi)-\frac{\cos (\chi) \sin (\varphi)}{\tan (\gamma)}\right) \\
\frac{\mathrm{d} \gamma}{\mathrm{d} r}= & \frac{\alpha \rho(r, \xi)}{2 \sin (\gamma)}-\frac{1}{v^{2} \tan (\gamma)}\left(g_{r}(r, \varphi)+\cos (\chi) \tan (\gamma) g_{\varphi}(r, \varphi)-\frac{v^{2}}{r}\right)+\frac{2 \omega \sin (\chi) \cos (\varphi)}{v \sin (\gamma)}+ \\
& +\frac{r \omega_{p}^{2} \cos (\varphi)}{v}\left(\frac{\cos (\varphi)}{\tan (\gamma)}+\cos (\chi) \sin (\varphi)\right) \\
\frac{\mathrm{d} \chi}{\mathrm{d} r}= & \frac{\sin (\chi) \tan (\varphi)}{r \tan (\gamma)}+\frac{2 \omega_{p}}{v}\left(\frac{\sin (\varphi)}{\sin (\gamma)}-\frac{\cos (\chi) \cos (\varphi)}{\cos (\gamma)}\right)+\frac{\sin (\chi)}{v^{2} \sin (\gamma) \cos (\gamma)}\left(r \omega_{p}^{2} \sin (\varphi) \cos (\varphi)-g_{\varphi}(r, \varphi)\right) \\
\frac{\mathrm{d} \beta}{\mathrm{d} r}= & 0 \\
\frac{\mathrm{d} \xi}{\mathrm{d} r}= & 0 \\
\frac{\mathrm{d} n}{\mathrm{~d} r}= & -\left[\frac{\partial \lambda^{\prime}}{\partial \lambda}+\frac{\partial \varphi^{\prime}}{\partial \varphi}+\frac{\partial v^{\prime}}{\partial v}+\frac{\partial \gamma^{\prime}}{\partial \gamma}+\frac{\partial \chi^{\prime}}{\partial \chi}+\frac{\partial \beta^{\prime}}{\partial \beta}+\frac{\partial \xi^{\prime}}{\partial \xi}\right] n
\end{aligned}\right.
$$

where $\lambda$ is the longitude, $\varphi$ is the latitude, $v$ is the velocity, $\gamma$ is the flightpath angle, $\beta=\left(m / C_{D} S\right)$ is the ballistic coefficient, $\alpha=\left(C_{L} S / m\right)$ is a modified lift coefficient, $\xi$ is an atmospheric correction coefficient, and $g_{r}$ and $g_{\varphi}$ are the radial and transversal components of the dimensions as it is for the case under examination. Several techniques exist to interpolate scattered data in one and two dimensions such as spline interpolation [40], multivariate polynomial [41,42], and radial basis function [43]. However, these techniques can be difficult to 
extend to arbitrary dimensions, or require regular grids. In this Paper, we propose reconstructing the density using linear interpolation based on the Delaunay triangulation [44] of the sampled points. We thus seek to approximate a multivariate function $f: X \rightarrow \mathbb{R}$ with $\boldsymbol{X} \subset \mathbb{R}^{d}$, where the elements of $\boldsymbol{X}$ are denoted by $\boldsymbol{x} \equiv\left(x_{1}, x_{2}, \ldots, x_{d}\right)$. We propose a simplicial-based interpolation given its possibility to be extended to arbitrary dimensions and its capability of allowing for the direct inclusion of derivative information to improve its accuracy [39]. Additionally, this methodology preserves the values at the nodes of the triangulation. This is important as it allows the conservation of a crucial information that is the value of the probability density at the sampled points as provided by the continuum-based propagation.

This section is structured as follows. Section III.A describes the linear interpolation methodology. Section III.B discusses the limitations of the Delaunay triangulation and proposes a different approach using $\alpha$ shapes. Section III.C proposes an improved interpolation methodology. Section III.C describes the procedure adopted to integrate the probability density and obtain the marginal distributions.

\section{A. Linear Interpolation}

As previously mentioned, the linear interpolation we adopt is based on a simplicial representation obtained through a Delaunay triangulation, which is unique for a given set of points. Given its heritage, uniqueness, and capability to be extended to arbitrary dimensions, it has been selected in this Paper to construct the simplical complex (i.e., the union of the simplices forming the triangulated state space) from the propagated scatter data. The construction of the Delaunay triangulation has been carried out using the Python package Delaunay of the scipy [45] library. Each of the data points in the considered state space (e.g., $r, v, \gamma$, and $\beta$ ) represents the coordinates of a vertex $V$ of the simplicial complex. At the same time, the probability densities relative to each vertex are the data values of the function $f$ to be interpolated. In general, the simplicial-based linear interpolation can be expressed as

$$
\boldsymbol{L}(\boldsymbol{x})=\sum_{i=1}^{N} \lambda_{i}(\boldsymbol{x}) f_{i}
$$

where $N$ is the number of vertices of the simplex (i.e., its dimensionality), $\boldsymbol{L}(\boldsymbol{x})$ is the linear interpolation at a point $\boldsymbol{x}$ inside the considered simplex, $\lambda_{i}(\boldsymbol{x})$ is the $i$ th barycentric coordinate of the point $\boldsymbol{x}$, and $f_{i}$ is the value of the function (the density in our case) at the $i$ th vertex. Once the barycentric coordinates of the vertices of the simplex are found, the linear interpolation of Eq. (8) can be performed. In other words, the linear interpolation is obtained through a weighted average of the value of the function at the simplex vertices, with the weights being the barycentric coordinates of the vertices (i.e., the distances of the vertices form the barycenter of the $d$-dimensional simplex).

\section{B. Alpha Shapes}

A drawback of Delaunay triangulation is that it is based on the convex hull of a set of points. Therefore, if the reconstructed shape is concave, additional unwanted triangles will be generated. For example, Fig. 1a shows a concave set of test points, which also contains a hole. It can be observed from Fig. 1b that the Delaunay triangulation generates simplices for the entire convex hull and fills the hole inside the set of points with additional simplices, which can compromise the accuracy of the interpolation.

Therefore, we introduce the concept of the $\alpha$ shape [38]. The $\alpha$ shape $\mathcal{C}_{\alpha}(V)$ of a set of points $V \subset \mathbb{R}^{d}$ is a subset of the Delaunay triangulation $\mathcal{D} \mathcal{T}(V)$. The objective of the $\alpha$ shape is to eliminate from the Delaunay triangulation all the excess simplices that are formed when a shape is not convex. As these simplices tend to be elongate, it is possible to use a test based on the radius of the circumhypersphere (the equivalent of the circum-radius in $d$ dimensions) $\sigma_{T}$ to prune triangles that are deemed too elongated by setting a threshold on the radius of the circum-hypersphere. Specifically, a simplex $\Delta_{T}$ belonging to the Delaunay triangulation also belongs to the $\alpha$ shape if 1) $\sigma_{T}<\alpha$ and the hypersphere of radius $\sigma_{T}$ is empty or 2) $\Delta_{T}$ is a face of another simplex in $\mathcal{C}_{\alpha}(V)$, where $\alpha$ is a hyperparameter, which has to be selected by the user. We present a possible way to select $\alpha$ in Sec. III.B.1. The first of the presented conditions is referred to as the alpha test. Following this definition and exploiting the properties of the Delaunay triangulation, it is possible to build the $\alpha$ shape through the following steps as described by Edelsbrunner [38]:

1) Compute the Delaunay triangulation of $V$, knowing that the boundary of the $\alpha$ shape is contained in it.

2) Determine $\mathcal{C}_{\alpha}(V)$ by inspecting all the simplices $\Delta_{T} \in \mathcal{D} \mathcal{T}(V)$; if the circum-hypersphere $\sigma_{T}$ is empty and $\sigma_{T}<\alpha$, we accept $\Delta_{T}$ as a member of $\mathcal{C}_{\alpha}(V)$, together with all its faces.

3) All $d$-simplices of $\mathcal{C}_{\alpha}(V)$ make up the interior of the $\alpha$ shape.

Given the aforementioned procedure, the $\alpha$ shape can be constructed starting from the Delaunay triangulation and removing all the simplices that do not pass the alpha test. For a $d$-simplex belonging to a Delaunay triangulation, the circum-hypersphere is empty by definition [44]; therefore, a simplex belongs to the $\alpha$ shape $\mathcal{C}_{\alpha}(V)$ if $\sigma_{T}<\alpha$. Therefore, the test only requires the computation of the radius of the circumhypersphere for a generic $d$-dimensional simplex that is [46]

$$
\sigma_{T}=R=\sqrt{-\frac{C \boldsymbol{M}_{11}^{-1}}{2}}
$$

Applying the $\alpha$-shape algorithm to the set of points of Fig. 1a with an $\alpha$ value of 0.25 , we get the $\alpha$ shape of Fig. 2, thus improving the shape reconstruction with respect to the Delaunay triangulation of Fig. 1b.

The interpolation using the Delaunay triangulation of Eq. (8) can be directly extended to the $\alpha$ shape, the only difference being that the simplices used are those belonging to the $\alpha$ shape and not to the Delaunay triangulation. To give an example of the linear interpolation using $\alpha$ shape, it is possible to associate a weight to each point of Fig. 1a. For example, we can assume that

$$
f\left(\boldsymbol{x}_{i}\right)=y_{i}^{2}
$$

To test the interpolation technique, we randomly select ten points from the set, remove them, and generate the $\alpha$ shape; we then interpolate the function at the coordinates of the ten test points (Fig. $\underline{3 a}$ ).
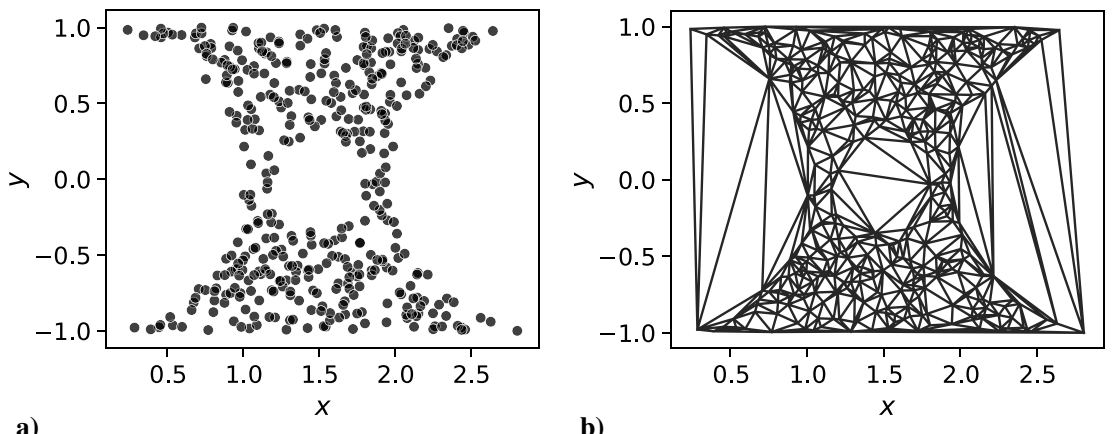

b)

Fig. 1 Example of Delaunay triangulation for a concave set of points. a) the set of scattered points; b) the resulting Delaunay triangulation. 


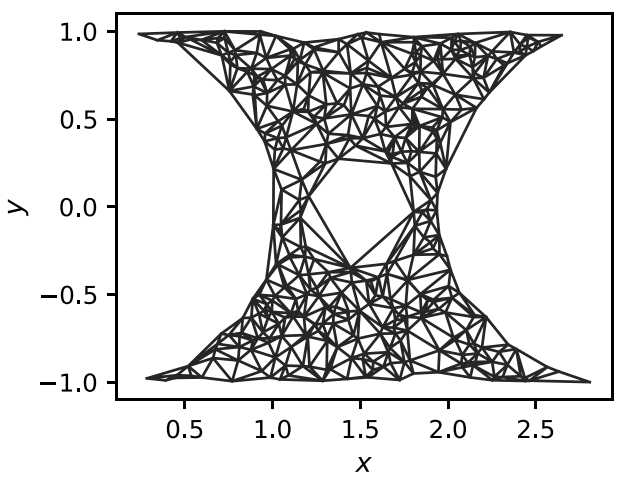

Fig. 2 Example of $\alpha$ shape for the set of test points.

Finally, we check the rms error between the interpolated values and the actual value of the function at these test points. Figure $3 \mathrm{~b}$ shows the results of this test for the ten test points, highlighting the rms percent error. The average error is $2.17 \%$, while the maximum is $11.89 \%$.

\section{Alpha Value Estimation}

The value of $\alpha$ in the construction of the $\alpha$ shape is a hyperparameter, which determines the simplices that will be removed from the initial Delaunay triangulation and, therefore, how closely the $\alpha$ shape will resemble the shape of the actual state space (Sec. III.B). To determine the $\alpha$ value, it is possible to perform a $k$-fold cross-validation, combined with a stochastic search algorithm. Specifically, we use a differential evolution (DE) search method [47] as it proved effective in the optimization of tuning parameters and hyperparameters [48]. In the $k$-fold cross-validation, the available set of points is subdivided into $k$ folds. One of the $k$ folds is then held out and used as validation set, while the other $k-1$ folds are combined into a training set. In this case, the points of the $k-1$ folds are used to construct the $\alpha$ shape: then, the interpolation at the points of the remaining fold is carried out. As we are interested in providing the best shape approximation for our dataset, we consider two conditions:

1) If any of the test points lies outside the $\alpha$ shape constructed with the training data, the value of $\alpha$ is rejected.

2) If all the test points are within the $\alpha$ shape, we compute the overall volume as the sum of the volumes of the simplices.

We repeat this procedure holding out a different fold each time. The output is then the average of the scores obtained for each fold. Throughout this Paper, the selected number of folds has been $K=5$. The result of the $k$-fold cross-validation depends on the value of $\alpha$; we apply a DE algorithm to find the value of $\alpha$ that minimizes the volume of the $\alpha$ shape computed following the previous points. Specifically, we use the differential evolution function of the Python scipy.optimize library [45]. Differential evolution is a stochastic direct search method in which a vector of parameters of size $N_{p}$, the population size, is evolved for a specified number of generations $N_{g}$ to find a global optimal solution. The initial population is randomly chosen inside the provided bounds, and a uniform distribution for the parameters in the search space is assumed. In our case, this translates into selecting a value of $\alpha_{i}$ inside the interval $\left(\alpha_{\min }, \alpha_{\max }\right)$. The new parameters vectors are generated using the bestlbin strategy [49]. For this Paper, the mutation rate has been set to $\eta_{m}=0.5$, and the recombination rate has been set to $\eta_{r}=0.7$, which are both typical values. The population size has been set to $N_{p}=40$, and the number of generations has been set to $N_{g}=60$. The presented strategy, however, can become computationally cumbersome when the dimension of the problem and/or the number of points increases. For example, obtaining the alpha values for the test case of Sec. IV.A takes around $60 \mathrm{~s}$ on the laptop used for the simulations in the Paper. This computational time can be too long with respect to the other operations performed. Possible mitigation strategies include but are not limited to: use of the same $\alpha$ value for several snapshots until the state space does not deform considerably; use of the $\alpha$ value of the previous snapshot as initial value for the current one to improve convergence; increase the parallelization; reduce the number of $k$ folds in the cross-validation. For the cases in which this procedure is deemed impractical, alternative procedures can be used to select the $\alpha$ value. For example, the distances between each point and its nearest neighbor can be evaluated. Then, the value of $\alpha$ can be selected as the average, maximum, minimum, or median value among these distances [50]. For example, the minimum value will tend to discard more elongated triangles but can also exclude parts of the tails of the distribution. The selection among this value can be performed by testing them on selected points, similarly to the cross-validation previously described. In addition, they can be used as starting values for the differential evolution algorithm.

\section{Gradient Enhanced Interpolation: Reduced Dual Taylor Expansion}

As shown in Fig. 3b, a direct application of the linear interpolation methodology can result in relative errors larger than $10 \%$ for selected points. We can expect that with an increase in the number of dimensions of the problem and complexity of the state space also the accuracy of the interpolation may degrade. An error in the interpolation directly maps into an error in the computation of the integral of the probability density and therefore worsens the approximation of the marginals, especially if large errors concentrate in high-density areas. Therefore, we propose a novel methodology that integrates supplementary derivative data into the presented interpolation [Eq. (8)] scheme to improve the accuracy of the approximation. The method we propose replaces the value of the function at the simplex vertices $f_{i}$ with its reduced dual Taylor expansion [39]. The procedure applies to all schemes that are based on function values at discrete nodes, provided the derivative data are available at the same nodes as the function values. This characteristic perfectly fits the interpolation procedure we propose, allowing for its extension and enhancement. The $n$ th-order reduced dual Taylor expansion of the $m$ th kind $\mathcal{D}_{x}^{m n}$ is defined as [39]

$$
\mathcal{D}_{x}^{m n}[f]:=\sum_{|\kappa| \leq n} \frac{1}{\kappa !} C_{|\kappa|}^{m n}(x-.)^{\kappa} f^{(\kappa)}(.)
$$

with

$$
C_{|\kappa|}^{m n}:=\left(\begin{array}{c}
m+n \\
m
\end{array}\right)^{-1}\left(\begin{array}{c}
m+n-|\kappa| \\
m
\end{array}\right)
$$

where $C_{|\kappa|}^{m n}$ are the reduction coefficients. The only difference between a standard dual Taylor expansion and Eq. (11) is exactly

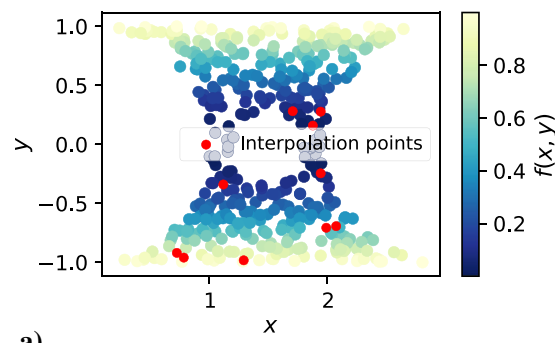

a)

Fig. 3 Interpolation example. a) interpolated function and selected inter

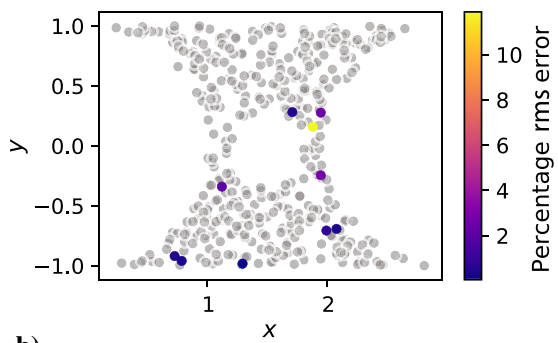

b) 
these coefficients. The modified expression can be used to raise the order of generic multivariate approximation schemes, provided they use a polynomial approximation of data at specified nodes and that the derivative information is available at these nodes. The implementation of Eq. (11) in our linear approximation scheme is straightforward: we just need to replace the input samples of $f$ with the corresponding samples of $\mathcal{D}_{x}^{m n}[f]$, which can be obtained using the supplementary derivative data. The interpolation scheme of Eq. (8) is then replaced by

$$
\tilde{\boldsymbol{L}}(\boldsymbol{x})=\sum_{i=1}^{N} \lambda_{i}(\boldsymbol{x}) \mathcal{D}_{x}^{m n}[f]\left(\boldsymbol{x}_{k}\right)
$$

The approximation order will then be raised from $m$ to $m+n$ [39]. The presented procedure perfectly adapts to the chosen methodology for the propagation of uncertainties (Sec. II). In fact, as we already compute the Jacobian of the dynamics, it is of little effort and computational cost to include the expressions for the derivative of the density with respect to the integration variables and return it as an additional output of the density propagation. Equation (14) provides an example for the dynamics of Eq. (ㅁ),

$$
\left\{\begin{array}{l}
\frac{\partial n}{\partial r}=-\left(\frac{v \sin \gamma}{r^{2}}-\frac{\partial g(r)}{\partial r} \frac{\cos \gamma}{v}-\frac{\partial \rho(r, \xi)}{\partial r} \frac{v}{\beta}\right) n \\
\frac{\partial n}{\partial v}=-\left(\frac{g(r) \cos \gamma}{v^{2}}-\frac{\sin \gamma}{r}-\frac{\rho(r, \xi)}{\beta}\right) n \\
\frac{\partial n}{\partial \gamma}=-\left(\frac{g(r) \sin \gamma}{v}-\frac{v \cos \gamma}{r}\right) n \\
\frac{\partial n}{\partial \beta}=-\frac{v \rho(r, \xi)}{\beta^{2}} n \\
\frac{\partial n}{\partial \xi}=-\frac{v}{\beta} \frac{\partial \rho(r, \xi)}{\partial \xi} n
\end{array}\right.
$$

With this procedure, we obtain at each node the required information for a direct application of Eq. (13). To demonstrate the improvement introduced by the gradient-enhanced interpolation, the test case of Fig. $3 \mathrm{a}$ is performed again, this time using the reduced dual Taylor expansion. For the function of Eq. (10), the derivatives with respect to $x$ and $y$ are

$$
\left\{\begin{array}{l}
\frac{\partial f}{\partial x}=0 \\
\frac{\partial f}{\partial y}=2 y
\end{array}\right.
$$

Including this information, we can perform the interpolation for the same test points. Figure $\underline{4}$ shows the resulting rms percent error, which is close to zero. The introduction of the derivative information has thus strongly improved the results of the linear interpolation. It has to be noted that this is a particular case: the interpolated function is quadratic [Eq. (15)], and with the reduced dual Taylor expansion,

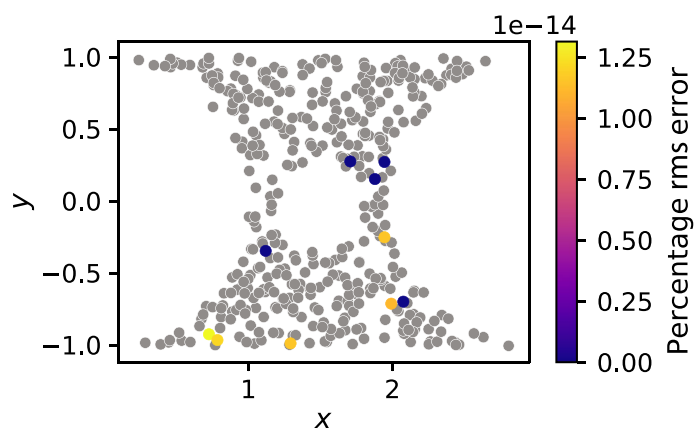

Fig. 4 RMS percent error for the interpolation enhanced by the reduced dual Taylor expansion. we introduce a second-order approximation, which matches the order of the function to be interpolated.

\section{Marginalization}

The density reconstruction using alpha shapes and the reduced dual Taylor expansion can be used to obtain the marginal densities of the relevant variables. In our application, this allows for a better understanding of the reentry under uncertainties, predicting how they influence the evolution in time of the reentry velocity, altitude, flight-path angle, and derived quantities such the dynamic pressure and heat rate. Given the generic $d$-dimensional state space $P_{s}=\left\{x_{1}, x_{2}, \ldots, x_{d}\right\}$, the one-dimensional and two-dimensional marginals can be computed as

$$
\begin{gathered}
m_{x_{m}}=\int_{P_{s} \backslash\left\{x_{m}\right\}} n\left(x_{1}, \ldots, x_{d}\right) d x_{1} \ldots d x_{m-1} d x_{m+1} \ldots d x_{d} \\
m_{x_{m} x_{n}}=\iint_{P_{s} \backslash\left\{x_{m}, x_{n}\right\}} n\left(x_{1}, \ldots, x_{d}\right) d x_{1} \ldots d x_{m-1} d x_{m+1} d x_{n-1} d x_{n+1} \ldots d x_{d}
\end{gathered}
$$

which is an integration of the probability density over all the dimensions in the state space, except the ones of the marginal axes. These expressions for the computation of the marginals apply to continuous functions. However, for the case under examination, only a discrete representation of the probability density is available through the interpolation procedure described in the previous sections. The computation of the marginals is here applied to the snapshots obtained through integration of Eq. (3), in other words, the scattered data in the sate space at each time step separately. As the procedure is similar for both one-dimensional and two-dimensional marginals, for a clearer explanation, we focus here on the computation of one-dimensional marginals. To do so, it is necessary to adapt Eq. (16) to scattered data. In addition, it is necessary to consider the nature of the data of the problem and, specifically, the evolution of the state space geometry with time. As shown for example by Fig. 6 , during the reentry process, the state space tends to suffer from a substantial deformation. We can thus obtain elongated state spaces, which are difficult to interpolate altogether, even when $\alpha$ shapes are employed. In addition, this strategy can reduce memory usage issues that can arise when generating the $\alpha$ shape in high dimensions. Consequently, we decided to overcome this issue by using a binning strategy to compute the marginals. First, we select the axis along which the marginal must be computed $x_{m}$. Then, this axis is subdivided into $N_{b}$ equally distributed bins, whose width is given by

$$
s_{b}=\frac{\max \left(x_{m}\right)-\min \left(x_{m}\right)}{N_{b}}
$$

In this way, slices of the original set of points are constructed to obtain a set $B$ containing the $N_{b}$ subsets of the scattered data in which $x_{m}$ is subdivided,

$$
B=\left\{\boldsymbol{x} \in V \mid x_{b, i} \leq x_{m} \leq x_{b, i+1} \forall i \in 1, \ldots, N_{b}\right\}
$$

where $x_{m}$ is the value of the $m$ th coordinate of the point $\boldsymbol{x}$ and $x_{b, i}$ is the coordinate of the $i$ th bin edge. For each subset of points contained in $B$, an $\alpha$ shape is constructed to perform the interpolation and compute the integral of the density relative to the considered bin. We then compute the marginal probability for each bin dividing the integral by the width of the bin so that the marginal is given by

$$
m_{x_{m}}=\left\{\frac{W\left(B_{i}\right)}{s_{b}} \forall B_{i} \in B\right\}
$$

where $W\left(B_{i}\right)$ is the integral of the probability density relative to the $i$ th set of points $B_{i}$ obtained as expressed by Eq. (19). At this point, it is important to outline the computational procedure for this integral. For the sake of clarity, let us consider a two-dimensional example for which 

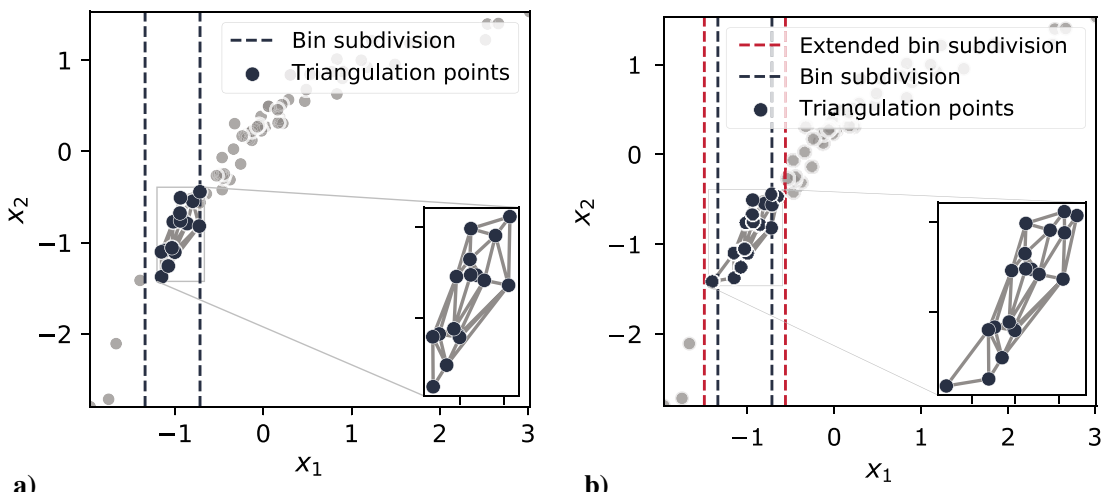

Fig. 5 Examples of $\alpha$-shape construction for a) a bin and b) an extended bin.

Fig. 5a shows a sample distribution of scattered data with an elongated state space. Suppose we are interested in the marginal distribution relative to $x_{1}$. For each bin subdivision, as highlighted in Fig. 5 a, an $\alpha$ shape is created using the points inside the bin. However, if we only use the points strictly contained inside the bin, the triangulation will exclude a portion of the volume of the state space, with a consequent underestimation of the integral. Therefore, each bin is expanded using buffer widths on both sides (Fig. 5b). In this way, the expanded bin $B_{i}^{\prime}$ allows for a triangulation, which fully includes the original bin.

Once this extended $\alpha$ shape has been created, the interpolation is performed. The interpolating points are the barycenters of the simplices of the $\alpha$ shape so that the integral of the density inside the extended bin is

$$
W\left(B_{i}^{\prime}\right)=\sum_{k=1}^{N_{s_{i}}} n\left(\boldsymbol{x}_{G_{k}}\right) v_{k}
$$

where $n\left(\boldsymbol{x}_{G_{k}}\right)$ is the interpolated value of the probability density at the barycenter of the $k$ th simplex $x_{G_{k}}$ as obtained using Eq. (13) of the gradient-enhanced interpolation scheme described in Sec. III.C, $N_{s_{i}}$ is the number of simplices contained inside the extended bin $B_{i}^{\prime}$, and $v_{k}$ is the volume of the $k$ th simplex. The integral computed using Eq. (21) refers to the extended bin. It is thus necessary to rescale this value. $\overline{\mathrm{We}}$ do not know with precision the volume of the state space contained inside the bin. However, it will be contained inside the volume of the $\alpha$ shape constructed with the initial bin $\mathcal{C}_{\alpha}\left(B_{i}\right)$ (Fig. 5a) and the one constructed with the extended bin $\mathcal{C}_{\alpha}\left(B_{i}^{\prime}\right)$ (Fig. 5b). Therefore, the integral is scaled with the average between these two volumes as follows:

$$
W\left(B_{i}\right)=W\left(B_{i}^{\prime}\right) \frac{\operatorname{Mean}\left(v_{\mathcal{C}_{\alpha}\left(B_{i}\right)}, v_{\mathcal{C}_{\alpha}\left(B_{i}^{\prime}\right)}\right)}{v_{\mathcal{C}_{\alpha}\left(B_{i}^{\prime}\right)}}
$$

Repeating this procedure for each bin in the set $B$ allows for the computation of the one-dimensional marginal. For the computation of the two-dimensional marginals, the procedure is analogous, with the binning performed over the two dimensions of the marginals $\left(x_{m}, x_{n}\right)$.

\section{Test Cases}

This section presents a series of relevant test cases to the problem under examination, applying the propagation methodology described in Sec. II and the reconstruction methodology outlined in Sec. III to planetary entry cases with different dynamical, gravitational, and atmospheric models. In this way, we show the applicability of the continuum propagation with models of different complexity. The results in terms of marginal distributions are compared with Monte Carlo simulations in terms of accuracy and execution time. In addition, for selected cases, the marginals of derived quantities of interest such as the dynamic pressure and the heat rate are computed, exploiting the already derived marginal distributions of the propagated variables. Finally, we use the derived marginals to assess the compliance of the mission to relevant constraints.

\section{A. Three-State Steep Earth Reentry}

The following section features a so-called strategic reentry [51], which represents a vehicle with a high ballistic coefficient on a steep, high-energy trajectory. The aim of these types of reentry is to more precisely pinpoint the impact location on Earth. The test case is performed using the three-state dynamics of Eq. (6). For the modeling of the atmosphere, a simple exponential model is adopted [Eq. (23)], while for the gravitational acceleration, an inverse square model is considered [Eq. (24)]. The expressions for the two models are

$$
\begin{gathered}
\rho(r)=\rho_{0} \exp \left\{\frac{H_{2}-\left(r-R_{p}\right)}{H_{1}}\right\} \\
g(r)=\frac{\mu_{p}}{r^{2}}
\end{gathered}
$$

where $\rho_{0}$ is a reference atmospheric density, $H_{1}$ and $H_{2}$ are constants related to the atmosphere of the planet, and $\mu_{p}$ is the gravitational parameter of the planet. The initial conditions of the reentry together with their uncertainties and the parameters used for the models are summarized in Table 1 . The uncertainties have been modeled with a Gaussian distribution so that the values of the initial conditions of Table $1, h_{0}, v_{0}, \gamma_{0}$, and $\beta_{0}$, represent the mean of a multivariate Gaussian.

For the case under examination, the uncertainties affect the initial state of the satellite at the reentry interface $r, v$, and $\gamma$ and the ballistic coefficient $\beta$. No uncertainty in the atmosphere is considered for this case, so the $\xi$ coefficient is neglected. We decided to consider the ballistic coefficient as it can influence the shape and evolution of the reentry trajectory and the prediction of the landing location. In addition, the exact quantification of the ballistic coefficient at reentry can

Table 1 Initial conditions and relevant parameter for the three-state strategic reentry on Earth

\begin{tabular}{lccc}
\hline \hline Parameter & Symbol & $\mu$ & $\sigma$ \\
\hline Initial velocity, $\mathrm{km} / \mathrm{s}$ & $v_{0}$ & 7.2 & 0.05 \\
Initial flight-path angle, deg & $\gamma_{0}$ & -30.0 & 0.1 \\
Initial altitude, $\mathrm{km}$ & $h_{0}$ & 125 & 2 \\
Ballistic coefficient, $\mathrm{kg} / \mathrm{m}^{2}$ & $\beta_{0}$ & 10,000 & 500 \\
Earth equatorial radius, $\mathrm{km}$ & $R_{p}$ & 6378.1 & -- \\
Earth gravitational parameter, $\mathrm{m}^{3} / \mathrm{s}^{2}$ & $\mu_{p}$ & $3.986 \times 10^{14}$ & - \\
Reference atmospheric density, $\mathrm{kg} / \mathrm{m}^{3}$ & $\rho_{0}$ & 1.215 & - \\
Atmospheric scale height, $\mathrm{km}$ & $H_{1}$ & 8.3 & - \\
Reference altitude, km & $H_{2}$ & 0 & -- \\
Lift-to-drag ratio & $C_{L} / C_{D}$ & 0 & - \\
\hline \hline
\end{tabular}


be difficult as it depends on the mass, cross-section, and drag coefficient of the spacecraft. The mass of the spacecraft at disposal may not be completely known, as the exact amount of residual propellant can be difficult to assess. The cross-sectional area of the satellite as well as even the exact value of the drag coefficient may be also uncertain. Following the procedure outlined in Sec. II, once the initial uncertainty distribution has been defined, this is sampled, and each sample is propagated using Eq. (6). In this case, we draw 750 samples. The result of the propagation using the continuity equation for selected snapshots (time instants, counted in seconds, after the starting epoch of the simulation) is summarized in Fig. 6.

The pair plot of Fig. $\underline{6}$ shows the projection of the sampled points for the snapshot at time $\bar{t}=30 \mathrm{~s}$. Along the main diagonal, the value of the density $n$ associated to each sample is presented for each variable: the diagonal plot in the first column shows the distribution of the samples in $r$ and the associated density value, the one in the second column refers to the velocity $v$, the one in the third column refers to the flight-path angle $\gamma$, and the one in the last column gives the density distribution as a function of the ballistic coefficient $\beta$. The off-diagonal two-dimensional plots show the relation between different couples of variables. The color map of the two-dimensional plots is associated to the magnitude of the density of each point. It is possible to observe how the state space, which started from a Gaussian structure, suffered a substantial deformation as the time passed. The deformation represents one of the challenges in reconstructing the probability density for the entire state-space volume.

\section{Comparison}

Figures $7 \mathrm{a}-7 \mathrm{c}$ show the one-dimensional marginals obtained with the density-based approach (DB) and the MC for the snapshot at time $t=24 \mathrm{~s}$. In this comparison, we have used 750 samples for the DB method and 750 (shaded histogram) and 5000 (dashed histogram) samples for the MC method.

To better compare the obtained marginal, we measure the difference between the distributions obtained with the DB and MC methods. To do so, we introduce two metrics commonly used in statistics to compare probability distributions that are the Hellinger distance $\Delta_{H}$ and the first Wasserstein distance $\Delta_{W}$. The Hellinger distance and the first Wasserstein distance between two PDFs $\mathcal{P}(x)$ and $\mathcal{Q}(x)$ are defined as

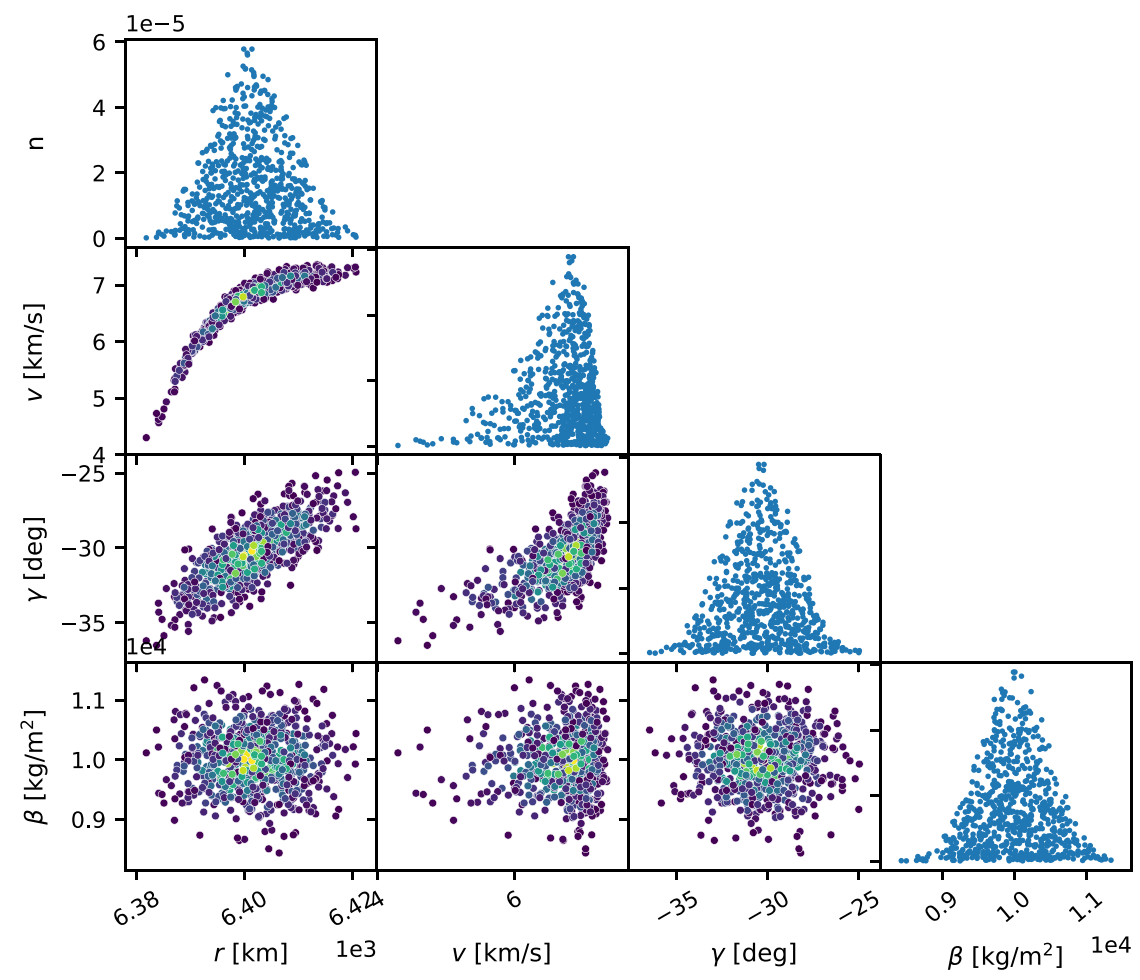

Fig. 6 Pair plot of the probability density propagation for the strategic reentry at two different snapshots at $t=30 \mathrm{~s}$.
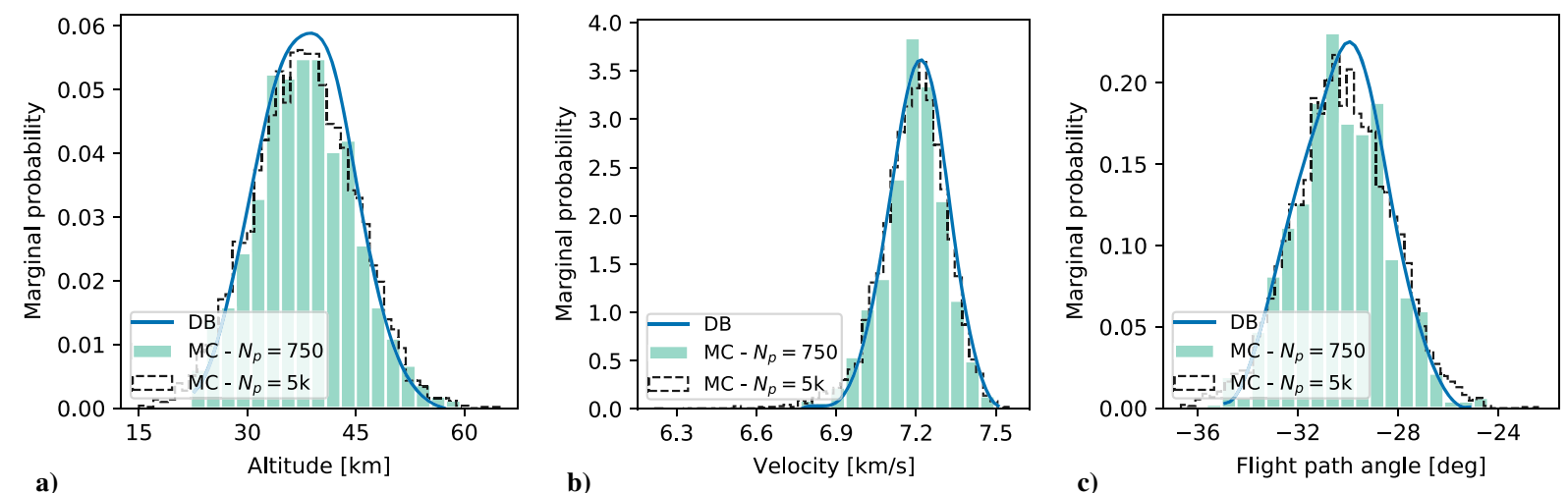

Fig. 7 DB vs MC one-dimensional marginals comparison at $t=24 \mathrm{~s}$ : a) altitude, b) velocity, and c) flight-path angle. DB: marginals with $750 \mathrm{samples.}$ MC: marginals with 750 and 5000 samples. 


$$
\begin{aligned}
& \Delta_{H}(\mathcal{P}, \mathcal{Q})=\sqrt{\frac{1}{2} \int_{-\infty}^{+\infty}(\sqrt{\mathcal{P}}-\sqrt{\mathcal{Q}})^{2} \mathrm{~d} x} \\
& \Delta_{W}(\mathcal{P}, \mathcal{Q})=\inf _{\pi \in \Gamma(\mathcal{P}, \mathcal{Q})} \int\|x-y\| \mathrm{d} \pi(x, y)
\end{aligned}
$$

where $\Gamma(\mathcal{P}, \mathcal{Q})$ is the collection of measures with marginals $\mathcal{P}$ and $\mathcal{Q}$. The Hellinger distance is a form of divergence measuring the difference between two distributions and always lies between 0 and 1 . The Wesserstein distance can be interpreted as the amount of work needed to transform one distribution into the other. Figures $8 \mathrm{a}$ and $8 \mathrm{~b}$ show the values of the Hellinger and Wesserstein distances, respectively, as a function of time (i.e., different snapshots fitting) for the variable $v$, comparing the DB and MC distributions with 750 to a reference distribution that is the MC marginal obtained with 5000 samples.

It is possible to observe that the DB method shows a better performance in both metrics from the initial states until about $28 \mathrm{~s}$ into the reentry. From that point on, the MC method performs slightly better. This is probably due to the characteristics of the simulations; in fact, after $30 \mathrm{~s}$, some of the samples reach the ground and are thus no longer used in the interpolation, and therefore a loss of performance can be expected (as shown by the Hellinger distance). On the other hand, it is surprising that the MC method considerably improves in both metrics when fewer points are available. The comparison after this instant might be less accurate, given that also the reference distribution obtained with 5000 samples suffers from a proportional reduction in the number of points available. Nonetheless, we show in Table 2, the comparison between the average value and the standard deviation over all the snapshots of the Hellinger and Wesserstein distances for $r, v$, and $\gamma$ for the DB and MC methods.

It can be observed that both metrics are, on average, lower for the DB method as opposed to the MC method for the same number of samples, and the standard deviation is also smaller in all instances, showing that the accuracy of the proposed method is competitive with $\mathrm{MC}$ using an equivalent sample size. A comparison of the computational effort for the strategic test case is presented in Table 3 for the DB case with 750 samples and the MC case with 750 and 5000 samples. The computational times have been subdivided into propagation time that is the time needed to propagate the trajectories and the marginalization time that is the time needed to reconstruct the marginal distribution from the scattered samples. The simulations
Table 3 Run-time comparison between the DB and MC methods for the strategic test case

\begin{tabular}{lccc}
\hline \hline Case & $\begin{array}{c}\text { Propagation } \\
\text { time, } \mathrm{s}\end{array}$ & $\begin{array}{c}\text { Marginalization } \\
\text { time, } \mathrm{s}\end{array}$ & $\begin{array}{c}\text { Total } \\
\text { time, } \mathrm{s}\end{array}$ \\
\hline $\mathrm{DB}(\mathrm{Np}=750)$ & 8.2 & 1.2 & 9.4 \\
$\mathrm{MC}(\mathrm{Np}=750)$ & 8.2 & 0.01 & 8.21 \\
$\mathrm{MC}(\mathrm{Np}=5000)$ & 74.0 & 0.06 & 74.06 \\
\hline \hline
\end{tabular}

were run on a Windows laptop equipped with an Intel Core i71065G7 @1.3 GHz processor and 16 GB of RAM.

Figure 9 shows a visual comparison between the two-dimensional marginals in altitude and velocity for two distinct snapshots $(t=8 \mathrm{~s}$ and $t=32 \mathrm{~s}$ ), obtained with the DB approach using 750 samples (Figs. 9a and 9d) and the MC approach using 750 and 5000 samples (Figs. 9b-9f), respectively.

A feature that can be observed in the obtained marginals (Figs. 7 and 9) is that the DB approach tends to produce more localized distributions. On one hand, the DB methodology uses the actual value of the probability density to obtain the marginals, while Monte Carlo estimates them and may underpredict or overpredict the distributions. However, the reconstructed marginals with the DB method are only as good as the fitting procedure used and the integration performed. The marginalization procedure proposed in Sec. III.C together with the interpolation presented in Sec. III.C introduces some simplifications and is limited to the envelope of the state space defined by the sampled points, which are then used to construct the $\alpha$ shape. This feature must be monitored as it may arise from a lower accuracy that can be obtained with the DB method at the boundaries of the distribution where a lower density of samples is available, an issue that might be mitigated by a more spatially efficient sampling technique.

Figure 10 shows the relative difference between the mean value predicted by the DB method with 750 samples and the MC method with 5000 samples at each snapshot for the three variables of interest $r, v$, and $\gamma$. It can be observed from Fig. 10 that the difference between the mean value as predicted by the $\mathrm{MC}$ and the $\mathrm{DB}$ methods remains below $1 \%$ for both the radius and flight-path angle, while it increases up to $8 \%$ for the velocity in the last part of the trajectory. This behavior confirms that in the last part of the trajectory, when fewer samples are available (in the last instant, only 110 samples out of 750 are available), the performance deteriorates as expected.
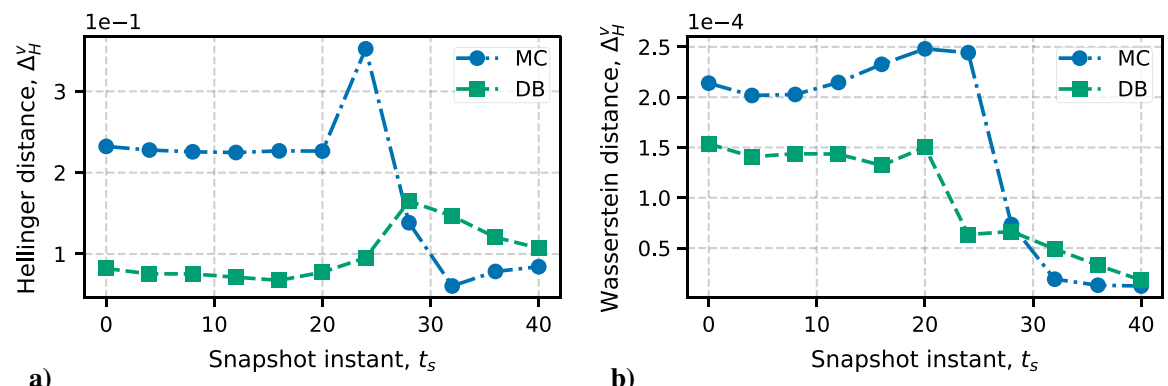

Fig. 8 Variation of a) the Hellinger distance and b) Wasserstein distance in time for the velocity. Both distances compare the 750 samples marginals of the DB and MC methods to the 5000 MC samples.

Table 2 Comparison of mean and standard deviation of the Hellinger and Wasserstein distances for the considered states

\begin{tabular}{lcccccc}
\hline \hline & $r$ & & & $v$ & & $\gamma$ \\
\hline Methodology & $\mu_{\Delta_{H}}$ & $\sigma_{\Delta_{H}}$ & $\mu_{\Delta_{H}}$ & $\sigma_{\Delta_{H}}$ & $\mu_{\Delta_{H}}$ & $\sigma_{\Delta_{H}}$ \\
DB & 0.1354 & 0.0327 & 0.0986 & 0.0313 & 0.0967 & 0.0064 \\
MC & 0.1679 & 0.0623 & 0.1889 & 0.0840 & 0.1195 & 0.0095 \\
Methodology & $\mu_{\Delta_{W}}$ & $\sigma_{\Delta_{W}}$ & $\mu_{\Delta_{W}}$ & $\sigma_{\Delta_{W}}$ & $\mu_{\Delta_{W}}$ & $\sigma_{\Delta_{W}}$ \\
DB & $3.12 \times 10^{-6}$ & $8.20 \times 10^{-7}$ & $9.95 \times 10^{-5}$ & $5.06 \times 10^{-5}$ & $4.80 \times 10^{-1}$ & $1.02 \times 10^{-1}$ \\
MC & $4.93 \times 10^{-6}$ & $1.32 \times 10^{-6}$ & $1.52 \times 10^{-6}$ & $9.52 \times 10^{-5}$ & $6.94 \times 10^{-1}$ & $1.64 \times 10^{-1}$ \\
\hline \hline
\end{tabular}




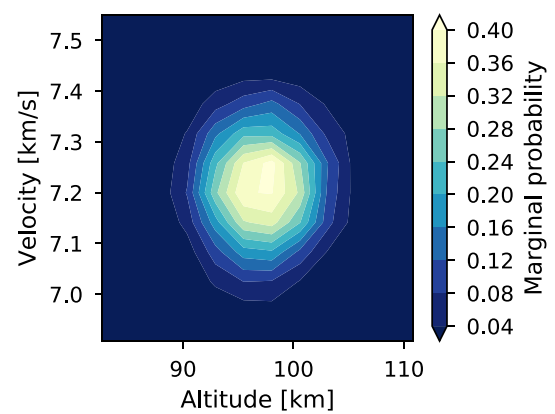

a) DB method with 750 samples

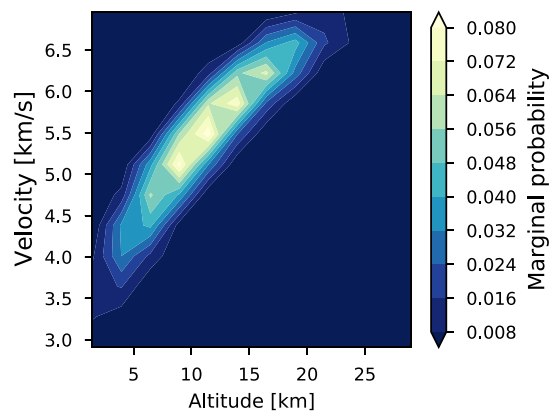

d) DB method with 750 samples

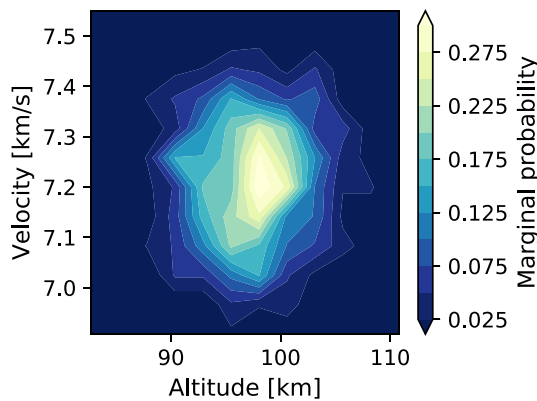

b) MC method with 750 samples

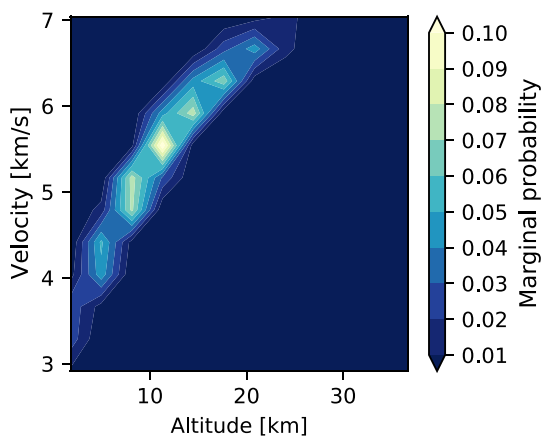

e) MC method with 750 samples

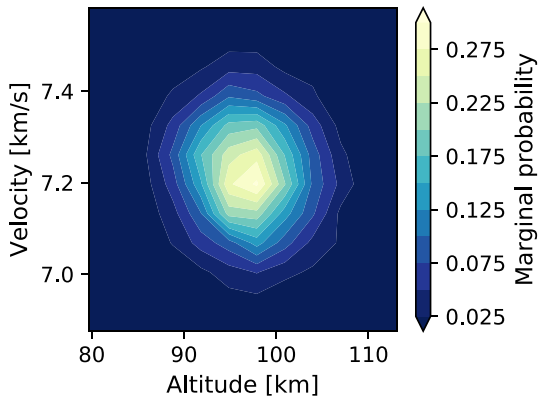

c) MC method with 5000 samples

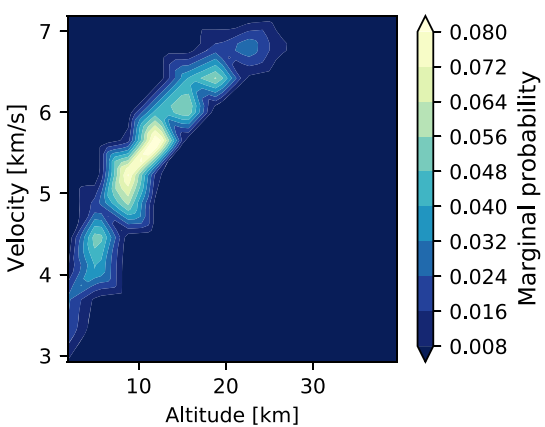

f) MC method with 5000 samples

Fig. 9 DB vs MC two-dimensional marginals in $h$ - $v$. Top row: snapshot time $t=8 \mathrm{~s}$. Bottom row: snapshot time $t=32 \mathrm{~s}$.

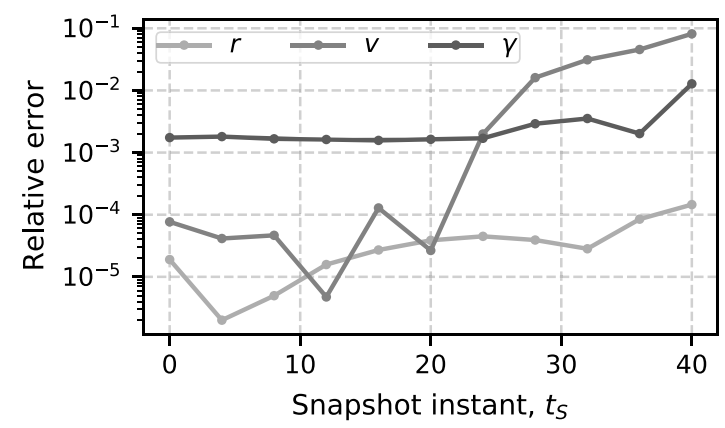

Fig. 10 Evolution of the relative difference between the expected value predicted by the DB method (750 samples) and MC method (5000 samples) for $r, v$, and $\gamma$.

Figure 11 shows instead the difference between the standard deviation predicted by the DB method with 750 samples and the MC method with 5000 samples for each snapshot. The results show that the standard deviation predicted by the DB method follows the one estimated with the MC method. It also confirms what could be observed in Figs. 7 and 9 with the DB method tendency to underpredict the tails of the distributions.

\section{Mechanical and Thermal Loads Compliance}

During reentry assessments, it is not only of interest to predict the uncertainties in the position and velocity of the spacecraft but also to understand how they transfer to other relevant quantities such as the dynamic pressure and the heat rate. We compute these quantities using the expressions

$$
\begin{aligned}
& \bar{q}=\frac{1}{2} \rho(h) v^{2} \\
& \dot{Q}=\bar{F}_{q} K \sqrt{\frac{0.3048}{r_{n}}} \sqrt{\frac{\rho(h)}{\rho_{S L}}}\left(\frac{v}{7924.8}\right)^{3.15}
\end{aligned}
$$

where $K=1.99876 \times 10^{8} \mathrm{~W} / \mathrm{m}^{2}$ is a constant, $r_{n}$ is the curvature radius at stagnation point in meters, $\rho_{S L}$ is the air density at sea level in kilograms/meters cubed, and $\bar{F}_{q}$ is an averaging factor that depends on the shape of the object, its motion, and the reentry regime.

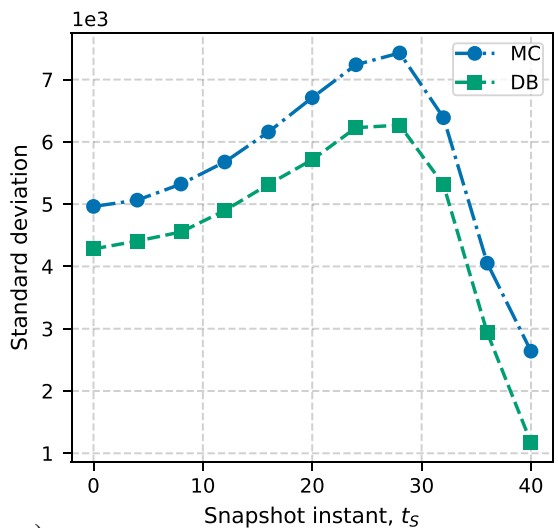

a)

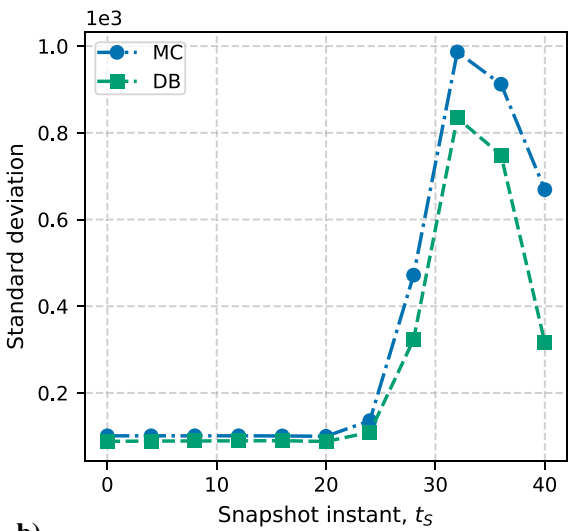

b)

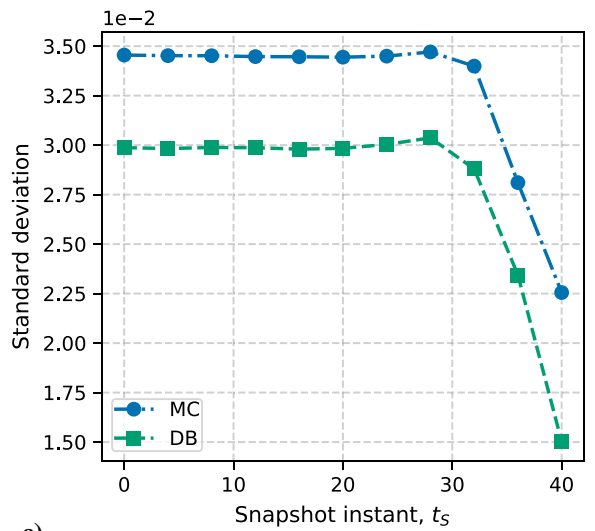

c)

Fig. 11 Evolution of the standard deviation predicted by the DB method (750 samples) and MC method (5000 samples) for a) $r$, b) $v$, and c) $\gamma$. 
The expression for the heat rate is a simplified version of the DetraKemp-Riddell correlation (DKR) [52], where we have omitted the term related to the stagnation point enthalpy. The DKR correlation is commonly used to predict the heat rate for hypersonic entries in Earth's atmosphere [13,53,54]. It is applicable to continuum flows so that it is less accurate at higher altitudes; however, for this demonstrative example, it has been used to compute the heat rate for the entire trajectory.

If we select the characteristics of the spacecraft, in other words, the curvature radius at the stagnation point and the averaging factor, both Eqs. (27) and (28) are only a function of the altitude and the velocity. We can thus exploit the previously computed marginal distributions in $h$ and $v$ to directly obtain information on the dynamic pressure and the heat rate during reentry. In fact, we can apply a transformation and scale the density accordingly. For example, in the case of the heat rate, we have

$$
\left\{\begin{array}{l}
\varphi(h, v)=v \\
\psi(h, v)=\bar{F}_{q} K \sqrt{\frac{0.3040}{r_{n}}} \sqrt{\frac{\rho(h)}{\rho_{S L}}}\left(\frac{v}{7924.8}\right)^{3.15}
\end{array}\right.
$$

When we apply this transformation, we need to scale the density accordingly, which can be accomplished by dividing the density in the original variables by the determinant of the Jacobian of the transformation as follows:

$$
n(v, \dot{Q})=n(h, v)\left|\begin{array}{ll}
\frac{\partial \varphi}{\partial h} & \frac{\partial \varphi}{\partial v} \\
\frac{\partial \psi}{\partial h} & \frac{\partial \psi}{\partial v}
\end{array}\right|^{-1}
$$
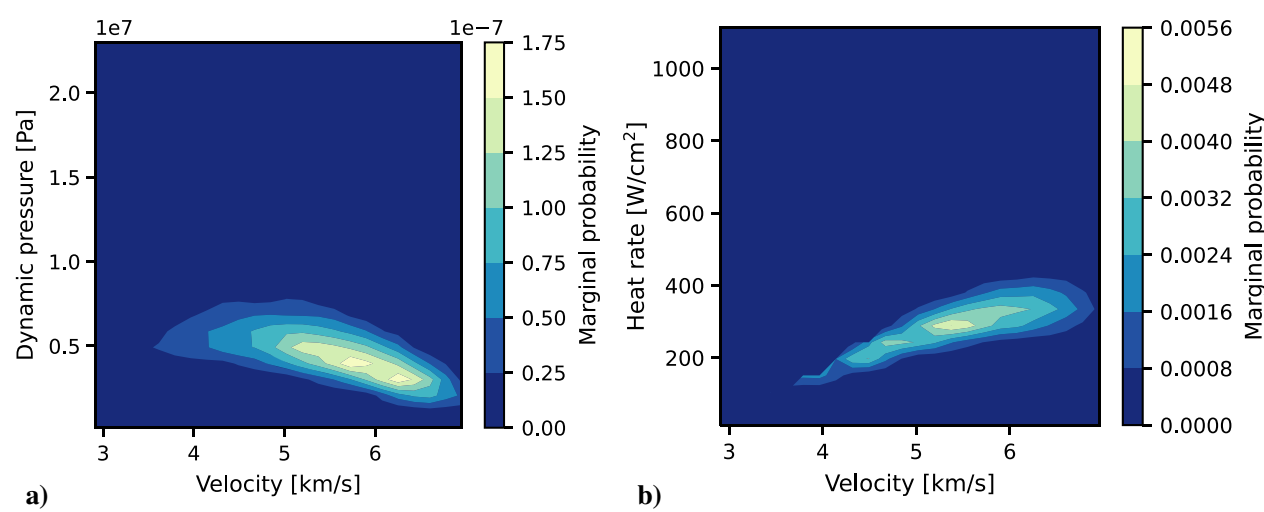

Fig. 12 Two-dimensional DB marginals of a) velocity vs dynamic pressure and b) velocity vs heat rate at time $t=32 \mathrm{~s}$.
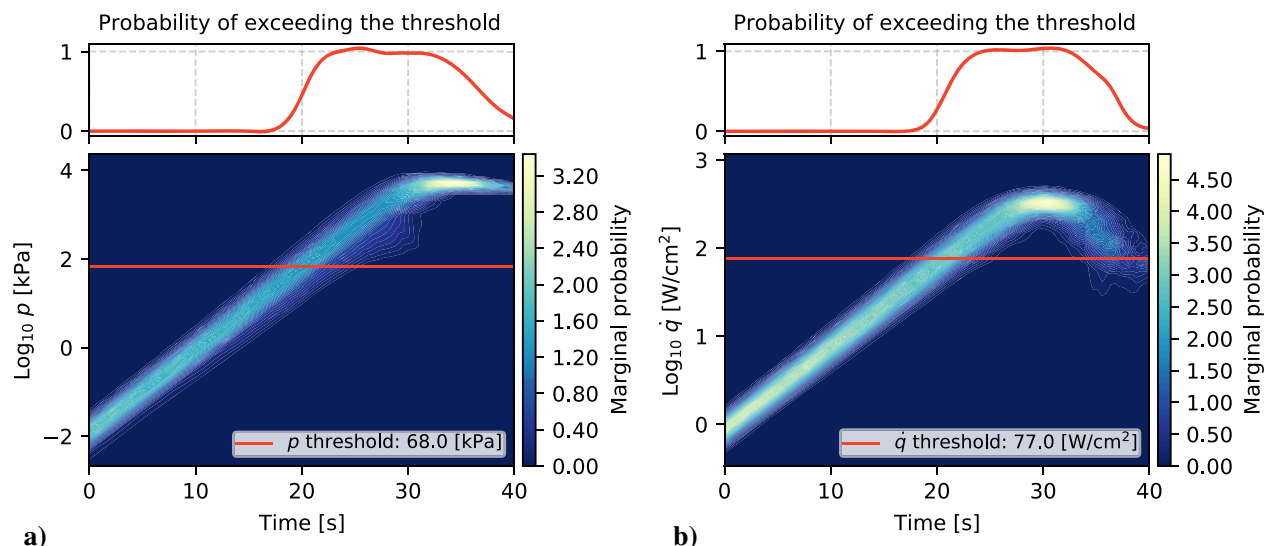

b)

With an equivalent procedure, we can also obtain the marginals for the dynamic pressure. Figures $12 \mathrm{a}$ and $12 \mathrm{~b}$ show examples for both the dynamic pressure and the heat rate for the test case under examination. The heat rate has been obtained considering a spherical shape with a $1 \mathrm{~m}$ radius. The corresponding averaging factor is $\bar{F}_{q}=0.234$ [14].

These two-dimensional marginals can then be integrated along $v$ to obtain the one-dimensional marginals for the dynamic pressure and heat rate. We can combine them together and obtain the evolution in time of the marginal probability for both the dynamic pressure and the heat rate. Figures $13 \mathrm{a}$ and $13 \mathrm{~b}$ show this time evolution. Each plot also features a threshold (in red). These values can be set by the user and defined to check different scenarios, for example, the limits in dynamic pressure and heat rate that a reentry capsule can withstand. For this test case, there is a threshold of $68 \mathrm{kPa}$ and $77 \mathrm{~W} / \mathrm{cm}^{2}$ [55] for the dynamic pressure and heat rate, respectively. The top part of the plot, represents the probability at each time step of crossing the limit. Initially, no part of the dynamic pressure and heat rate distributions crosses the thresholds. As time passes, the distributions shift toward higher dynamic pressures and heat rates so that parts of the distribution cross the threshold, increasing the probability. In the final states, the probability density starts to reduce again. This happens because some of the initial samples reach the ground before others and when they do their contribution to the total probability weight is removed.

\section{B. Six-State Steep Earth Reentry}

The example presented in the following section is an extension of the test case in Sec. IV.A in which a six-state propagation is used instead [Eq. (7)] and the gravity and atmosphere models have an increased complexity. The gravitational model used takes into account the earth oblateness $\mathbf{J} 2$ effect [56], and the atmospheric model is the 1976 U.S. Standard Atmosphere [57]. To use the U.S. Standard Atmosphere model into the continuity equation, a spline interpolation

a)

Fig. 13 Marginal evolutions in time: a) dynamic pressure and b) heat rate. In red, specific thresholds. On top, the probability in time of crossing the threshold. 
Table 4 Mean and standard deviation for the initial conditions of the augmented state space and values of the relevant parameters

\begin{tabular}{lccc}
\hline \hline Parameter & Symbol & $\mu$ & $\sigma$ \\
\hline Initial longitude, $^{\circ}$ & $\lambda_{0}$ & 0 & 0.2 \\
Initial latitude, $^{\circ}$ & $\varphi_{0}$ & 0 & 0.2 \\
Initial velocity, $\mathrm{km} / \mathrm{s}_{\text {Initial flight-path angle, deg }}$ & $v_{0}$ & 7.2 & 0.036 \\
Initial heading angle, deg $_{\text {Atmospheric correction }}$ & $\gamma_{0}$ & -30 & 0.15 \\
Initial altitude, $\mathrm{km}^{\text {Ballistic coefficient, } \mathrm{kg} / \mathrm{m}^{2}}$ & $\xi$ & 45 & 0.225 \\
Lift coefficient, $\mathrm{m}^{2} / \mathrm{kg}$ & $\chi_{0}$ & 125 & 0.05 \\
\hline \hline
\end{tabular}

of the density has been performed using the UnivariateSpline class of the scipy Python package. The UnivariateSpline class has the advantage of providing the derivative information that can be used directly into the equations of motion when needed. As previously mentioned, the uncertainties in the atmosphere can be included in the continuum approach. The procedure adopted here is to introduce a correction coefficient to scale the value of the density provided by the selected model as

$$
\rho(r, \xi)=\xi \hat{\rho}_{\mathrm{std} 76}(r)
$$

where $\xi$ is the atmospheric correction coefficient and $\hat{\rho}_{\text {std76 }}(r)$ is the spline representation of the atmospheric density as provided by the 1976 U.S. Standard Atmosphere model. More complex models can be considered, which include more than one uncertain parameters. The models must be represented with differentiable functions and all the uncertain parameters must be included in the augmented state space. For the case under examination, the uncertainties have been considered in all the initial states $\lambda, \varphi, v, \gamma$, and $\chi$ and in the atmospheric correction coefficient $\xi$, while no uncertainty in the ballistic coefficient has been included. Table 4 summarizes the mean value and

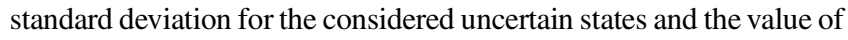
the remaining parameters necessary for the reentry simulation.

As is possible to observe, in this case, the uncertainties in the initial conditions have a smaller and more realistic standard deviation with respect to the ones presented in Table 1 . The largest uncertainty is represented by the atmospheric correction coefficient, which introduces a $5 \%$ standard deviation in the value of the atmospheric density. The uncertainty in the radial position has been removed as it is now the independent variable of the integration. This can allow setting a radius (altitude) value for the reentry interface and exclude the radius from the uncertainties. The presented test case is intended to show the applicability of the continuum propagation to a realistic reentry scenario, which considers the full dynamical representation, includes more realistic and accurate gravitational and atmospheric models, and takes into account the uncertainty in the atmospheric properties. The six-dimensional distribution of the initial uncertainty is then sampled, and each sample is propagated until the surface of the Earth is reached. Figure 14 shows the projections of the distribution of 1000 sampled points at the end of the propagation (Earth impact). For the sake of readability, the distribution in the atmospheric correction coefficient $\xi$ is omitted; however, as it is a coefficient, its derivative is zero [Eq. (7)], and the distribution at the final instant is the same as the initial one. On the main diagonal, we can observe the magnitude of the probability density associated to each sampled point.

It is interesting to observe how the distribution for this six-state variation of the strategic test case is considerably more regular and maintains a more Gaussian-like behavior throughout the reentry process when compared to its three-state counterpart (Fig. 6). The difference is due to the different uncertainties in the initial conditions, which are much more pronounced for the test case in Sec. IV.A. The different independent variable (from $t$ to $r$ ) may also have contributed to the different shape of the state space.

\section{Comparison}

Figure 15 shows the comparison between the one-dimensional marginals obtained with the DB and the MC methods for the relevant state variables. The comparison is between the $\mathrm{DB}$ marginals obtained with 1000 samples and the corresponding MC marginals obtained with 1000 (the green shaded histogram) and 50,000 samples (the black dashed histogram) for the final propagation instant, which correspond to landing.

The marginals show a similar behavior with respect to their threestate counterparts (Fig. 7): the distributions obtained with 1000 Monte Carlo samples show a more erratic behavior for all the states, especially in the areas around of the peak of the distribution, while the

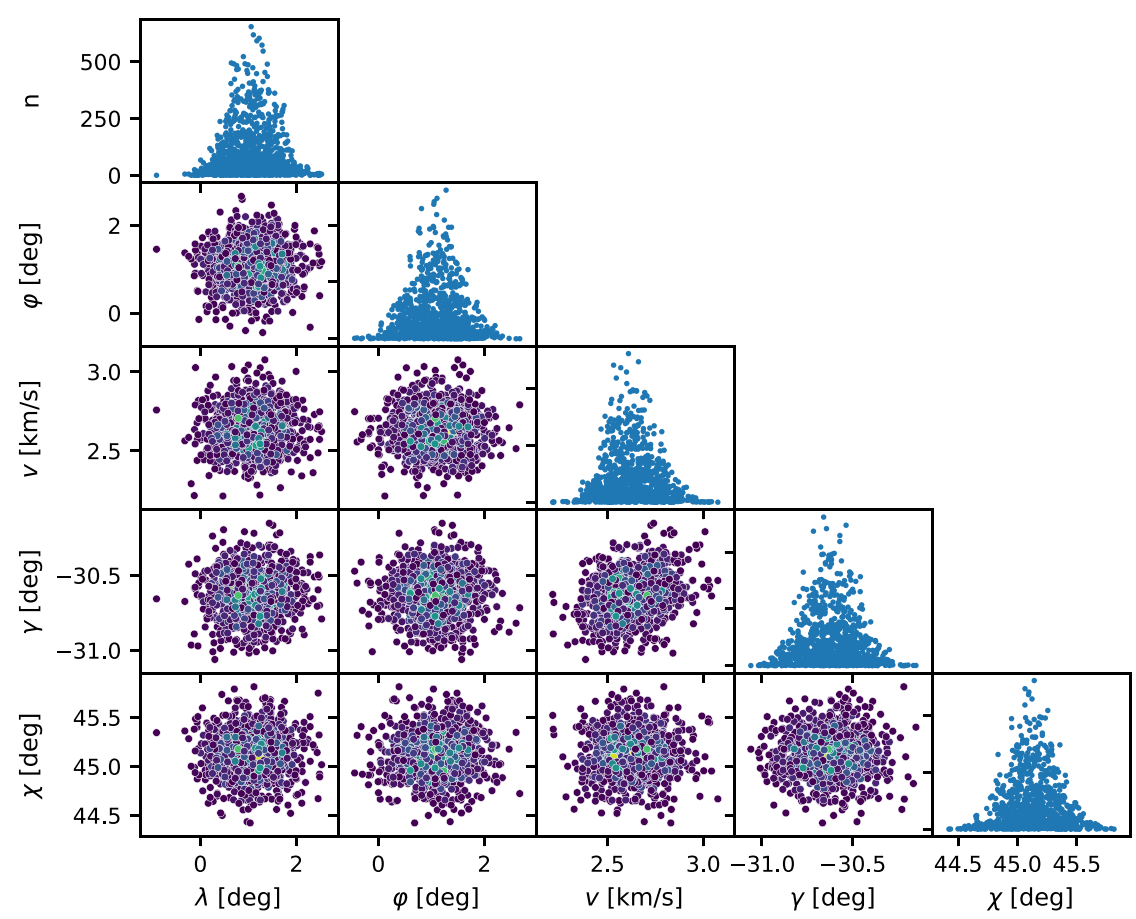

Fig. 14 Landing snapshot for the six-state steep entry test case. 


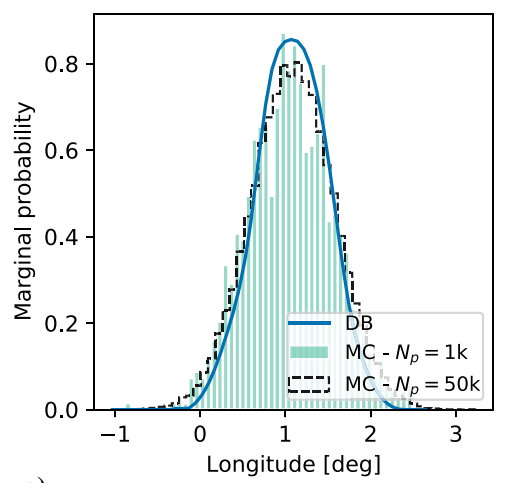

a)

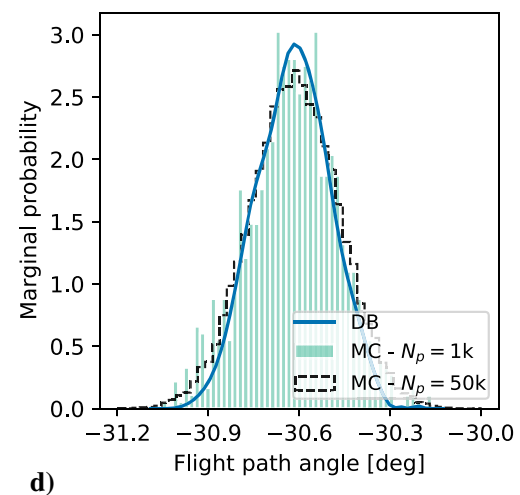

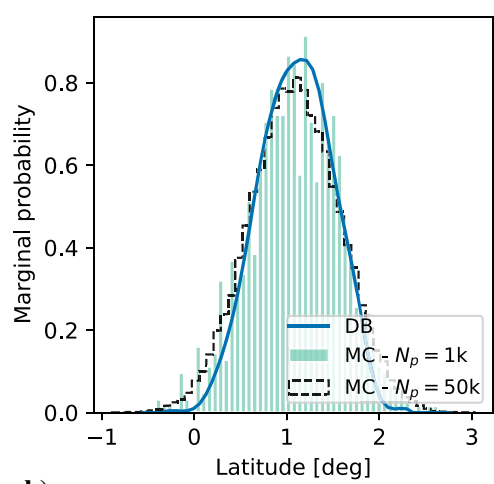

b)

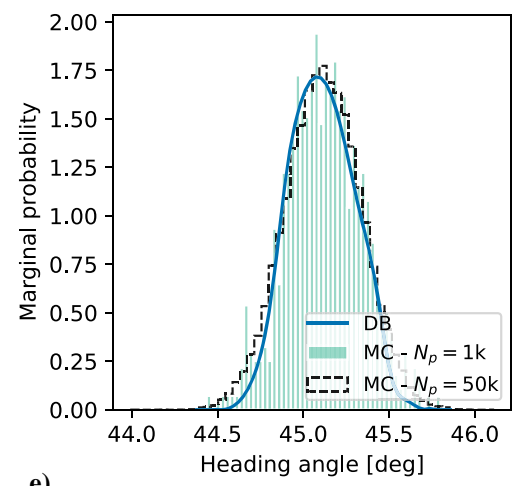

Fig. 15 DB vs MC one-dimensional marginals comparison at landing instant for 1000 samples and 50,000 samples.

corresponding DB marginals show a more regular behavior. However, even in this case, it is possible to observe the feature of the DB marginals when compared to the 50,000 Monte Carlo that is the presence of peaks and smaller tails, which in turn results in a smaller standard deviation in the prediction of the states at the landing instant. To better compare the quality of the distribution reconstruction with both the DB and the MC methods, a comparison using the metrics introduced in Sec. IV.A (Hellinger distance and Wasserstein distance) is performed. Figure 16 shows this comparison for three selected states (latitude, velocity, and heading angle). In the comparison, the distances have been computed taking as reference distribution the Monte Carlo simulation with 50,000 samples. In case of the DB methodology, the results for 50,000 samples are not present as the memory requirements were too high for the laptop used in this Paper.

The results of the comparison show that the marginals obtained with the DB method consistently perform better with respect to their MC counterparts with the same number of samples according to both the proposed metrics. However, some notable features can be identified: the Hellinger distance shows in all three comparisons a worsening in the performance of the DB method when passing from 5000 to 10,000 samples. This feature is confirmed also by the Wasserstein distance for the latitude marginal. The reason behind this behavior probably resides in the methodology used to obtain the marginal distribution as outlined in Sec. III.C when combined with the increasing requirements for computational resources due to the growing number of points; in fact, to limit the memory usage, a higher number of bins has been used, leading to a lesser performance in the estimation of the marginals. On the other hand, the Wasserstein distance (Figs. 16b,16d and 16f) shows that the DB marginals obtained with 1000 samples have a better or comparable result with respect to the $10,000 \mathrm{MC}$ simulation. A similar trend can be observed in two out of three comparisons of the Hellinger distance, where the heading angle case has a more pronounced difference between the $1000 \mathrm{DB}$ and the 10,000 MC simulations. Another interesting aspect, when comparing the results of Fig. 16 with the results of the previous test case Fig. 8 is that in the last instant of the six-state case the DB method still performs better then the MC, while for the three-state case, it has a worse performance. This feature is probably related to the fact the in this case, by introducing the radius as an independent variable, all the samples are maintained in the snapshot until the end, while in the case of Sec. IV.A, they are removed when they reach the ground, thus reducing the number of samples available for the fitting. A final comparison is provided for the run time of the code as a function of the number of points as shown in Fig. 17. The run time has been normalized with respect to the run time of the $50,000 \mathrm{MC}$ simulations so that all the other figures are a fraction of this reference time. Similarly to Sec. IV.A.1, the run times include the propagation time of the trajectory and the fitting time. As expected, for the same number of points, the DB method is always more time consuming as the fitting procedure requires more time than the histogram generation. However, it is also possible to observe that the DB method with 1000 samples requires about $1 / 4$ and $1 / 7$ of the time required by the 5000 and 10,000 sample MC methods, respectively, with a performance that is comparable to or worse than the one of the DB method, as shown in Fig. 16. In addition, Fig. 17 shows the ratio between the marginalization time and the propagation time for the DB method, which oscillates between 40 and $60 \%$.

\section{Six-State Mars Reentry}

The third test case features a six-state Mars reentry, whose propagation is again performed using Eq. (7). The initial conditions and the other relevant reentry parameters are based on the Mars Science Laboratory (MSL) mission [26,58], and they are summarized in Table 5. The modified lift coefficient, $\alpha=0.001 \mathrm{~m}^{2} / \mathrm{kg}$, corresponds to a $\overline{C_{L}}=0.18$ of a spacecraft with mass $m=2200 \mathrm{~kg}$ and crosssection $S=12.9 \mathrm{~m}$ [58].

As is possible to observe, in this case, we have considered a lifting entry with Gaussian uncertainties in the initial longitude, latitude, velocity, and flight-path angle together with the ballistic coefficient and the atmospheric correction coefficient. The gravity model for Mars is an inverse exponential in this case, equivalent to the one adopted in Sec. IV.A. The atmospheric density as a function of the altitude has been obtained from the curve fitting of the MSL data and has the expression [26]

$$
\rho(h)=\exp \left\{c_{0}+c_{1} h+c_{2} h^{2}+c_{3} h^{3}+c_{4} h^{4}\right\}
$$




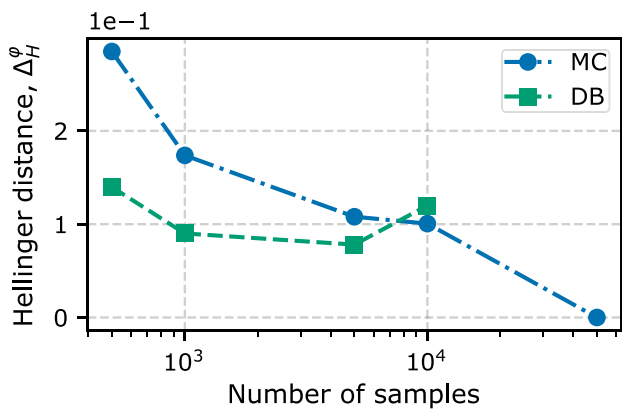

a) Hellinger distance for $\varphi$

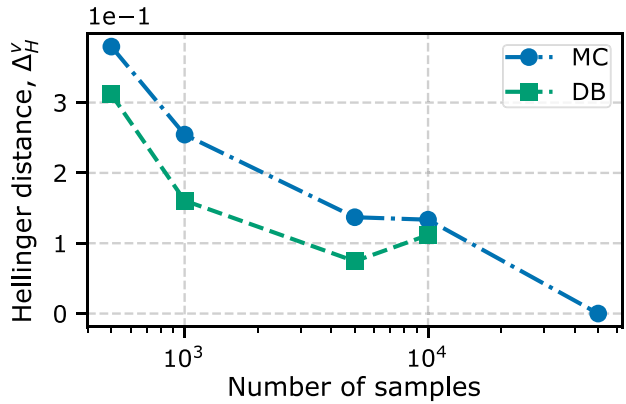

c) Hellinger distance for $v$

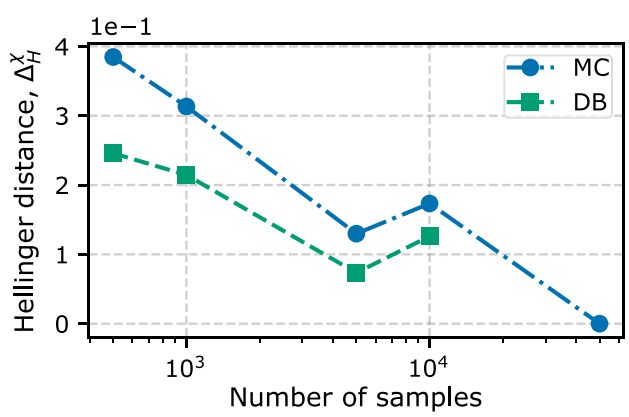

e) Hellinger distance for $\chi$

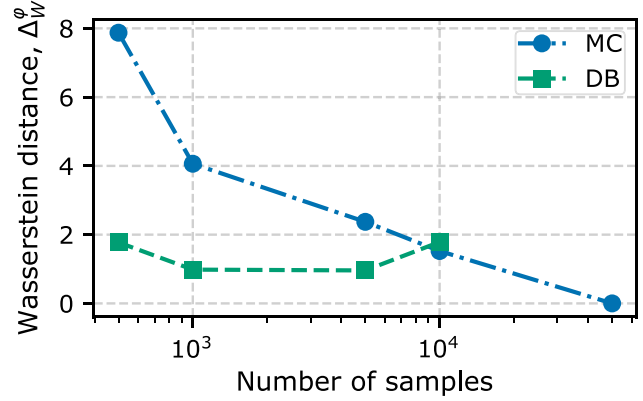

b) Wasserstein distance for $\varphi$

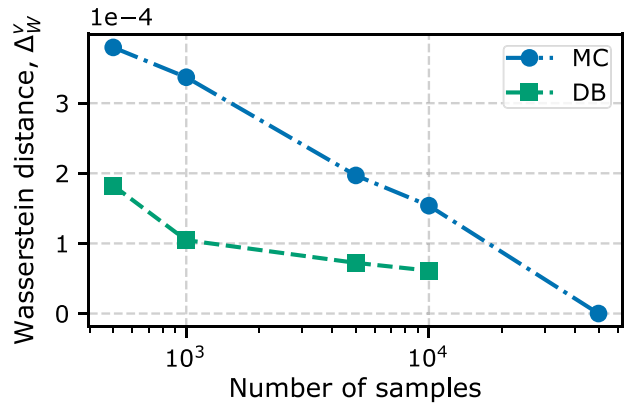

d) Wasserstein distance for $v$

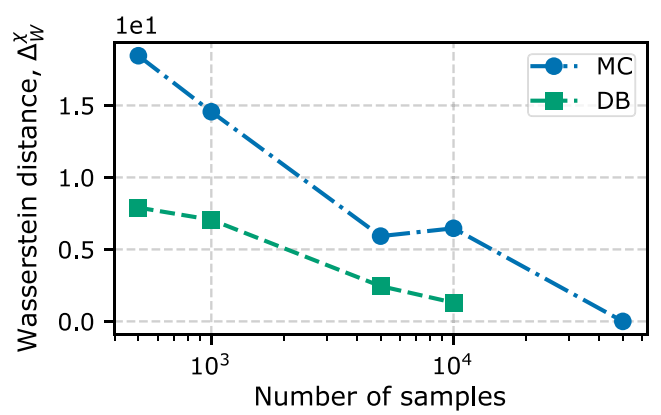

f) Wasserstein distance for $\chi$

Fig. 16 Variation of the Hellinger and Wasserstein distances between the DB and MC methods as a function of the number of samples for the landing snapshot.

where $c_{0}=-4.343, c_{1}=-9.204 \times 10^{-5}, c_{2}=-1.936 \times 10^{-11}$, $c_{3}=-7.507 \times 10^{-15}$, and $c_{4}=4.195 \times 10^{-20}$. Again, the uncertainty in the atmospheric density is implemented following the procedure outlined by Eq. (31), introducing the atmospheric correction coefficient $\xi$. Figure 18 shows the projections of the snapshot at the landing instant for the Mars test case, where we have excluded the ballistic coefficient for a better readability. We can observe that, differently from the previous test case (Fig. 14), the effect of the nonlinear dynamic is more pronounced and this translates into a more deformed state space, particularly in the coupling between the velocity,

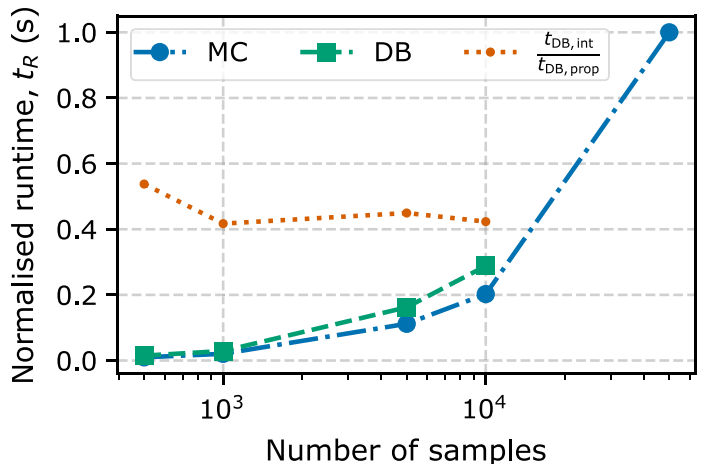

Fig. 17 Run-time comparison between the DB and MC methodology. In orange, the fraction between the interpolation and the propagation times for the DB method. the flight-path angle, and the atmospheric correction coefficient (the ballistic coefficient has a similar dependency).

\section{Comparison}

Figure 19 show the one-dimensional marginals obtained with the $\mathrm{DB}$ and $\mathrm{MC}$ methods for the velocity and the flight-path angle at the landing instant. The DB marginal has been obtained with 1000 samples, while the MC method shows the results obtained with

Table 5 Mean and standard deviation for the initial conditions of the augmented state space and values of the relevant parameters

\begin{tabular}{|c|c|c|c|}
\hline Parameter & Symbol & $\mu$ & $\sigma$ \\
\hline Initial longitude, ${ }^{\circ}$ & $\lambda_{0}$ & -90.07 & 0.5 \\
\hline Initial latitude, ${ }^{\circ}$ & $\varphi_{0}$ & -43.9 & 0.5 \\
\hline Initial velocity, $\mathrm{km} / \mathrm{s}$ & $v_{0}$ & 5.505 & 0.004 \\
\hline Initial flight-path angle, deg & $\gamma_{0}$ & -14.15 & 0.023 \\
\hline Ballistic coefficient, $\mathrm{kg} / \mathrm{m}^{2}$ & $\beta$ & 125.0 & 3.5 \\
\hline Atmospheric correction & $\xi$ & 1 & 0.05 \\
\hline Initial altitude, $\mathrm{km}$ & $h_{0}$ & 126 & - - \\
\hline Initial heading angle, deg & $\chi_{0}$ & 4.99 & - - \\
\hline Lift coefficient, $\mathrm{m}^{2} / \mathrm{kg}$ & $\alpha$ & 0.001 & - - \\
\hline Mars equatorial radius, $\mathrm{km}$ & $R_{p}$ & 3397 & -- \\
\hline Mars gravitational parameter, $\mathrm{m}^{3} / \mathrm{s}^{2}$ & $\mu_{p}$ & $4.283 \times 10^{13}$ & - - \\
\hline Mars rotational rate, $\mathrm{rad} / \mathrm{s}$ & $\omega_{p}$ & $7.095 \times 10^{-5}$ & -- \\
\hline
\end{tabular}




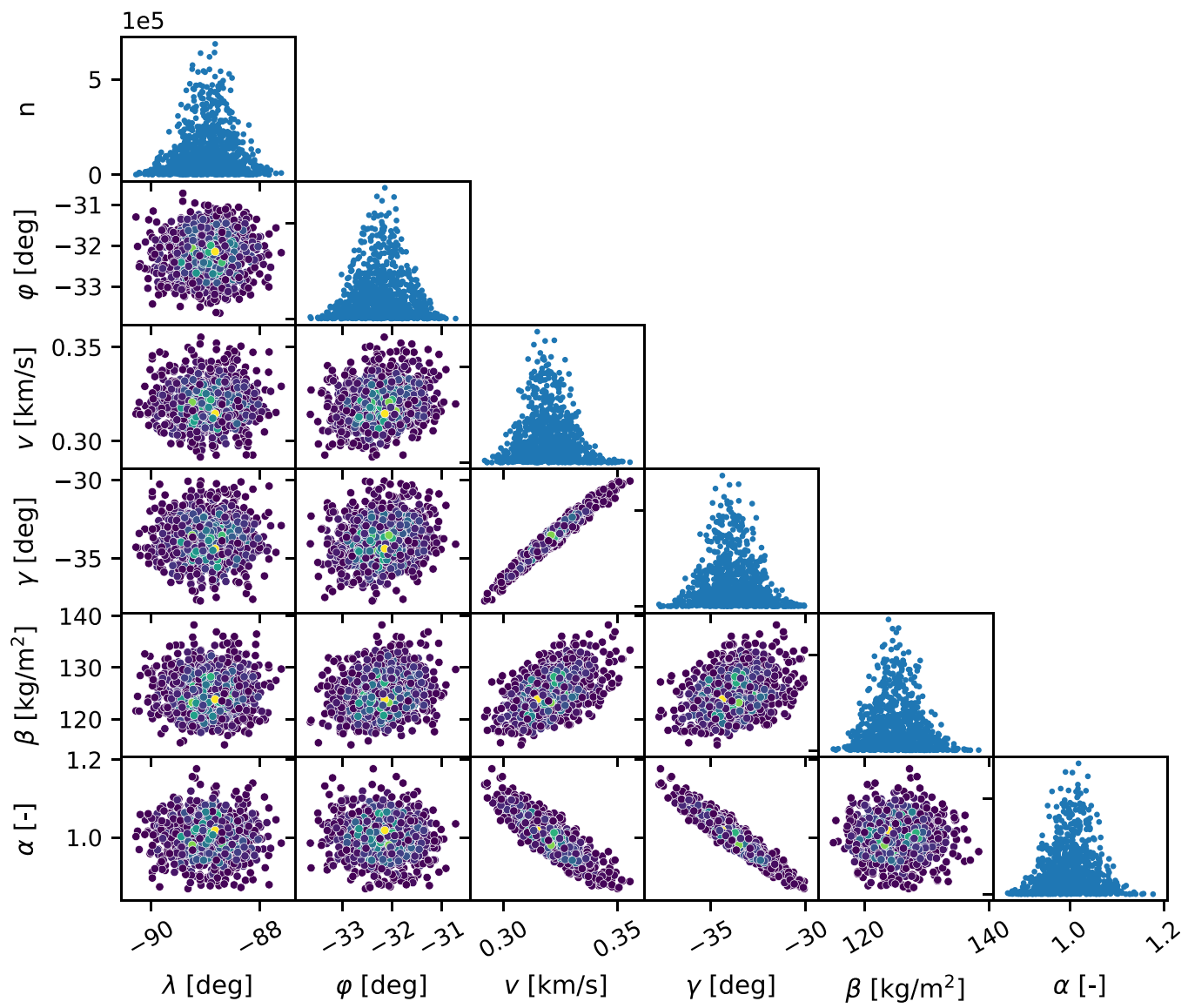

Fig. 18 Pair plot of the landing snapshot for the six-state unguided Mars entry test case.
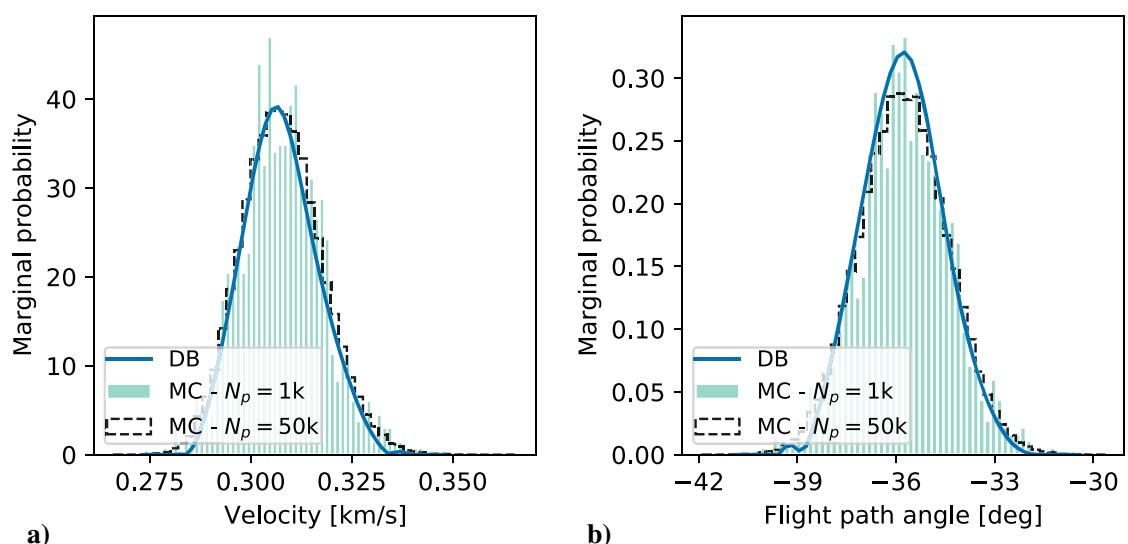

Fig. 19 DB vs MC one-dimensional marginals comparison at landing instant for the Mars reentry test case: a) velocity marignal and b) flight-path angle. DB method with 1000 samples and MC method with 1000 and 50,000 samples.

1000 samples (green shaded histogram) and 50,000 samples (black dashed histogram). The results show a good agreement of the DB method with the 50,000 samples MC simulation taken as a reference. However, one can still see the discrepancy around the tails of the distribution, with a more erratic behavior and a tendency to reproduce lower values of the marginal probability, as already seen in Figs. 7 and 15 . Figure 20 instead shows the comparison between the twodimensional marginals obtained with the DB and MC methods for the landing location coordinates expressed in longitude and latitude.

A quantitative comparison between the obtained marginals is again performed exploiting the metrics introduced in Sec. IV.A, in other words, the Hellinger and Wasserstein distances. The comparison is performed by computing the average and standard deviation of the Hellinger and Wasserstein distances over time for different numbers of sample points. The distances are computed, taking as reference the results obtained with the MC simulation with 50,000 samples. Figure 21 shows this comparison for the velocity and the flight-path angle as a function of the number of points. The solid lines represent the mean value, and the shaded area represents the standard deviation. The Hellinger distance (Figs. 21a and 21c) shows a regular behavior in both the considered variables for the DB method, with a constant improvement in performance when going from 500 to 5000 points. A worsening of the approximation happens instead for 10,000 points, as was the case for the Earth reentry test case (Fig. 16). In addition, it is possible to observe that, on average, the DB method shows a better performance with respect to the MC method, except for the 10,000 samples case, and a smaller standard deviation. The Wasserstein distance (Figs. 21b and 21d) shows a similar behavior 


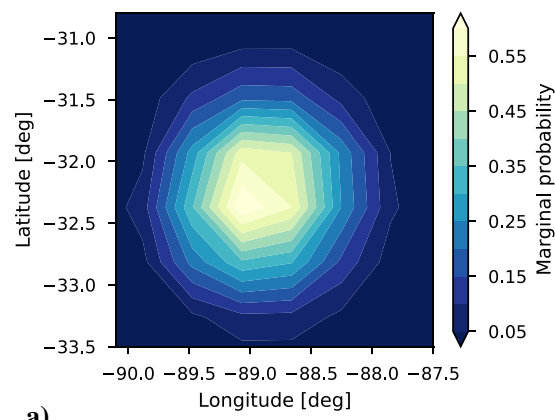

a)

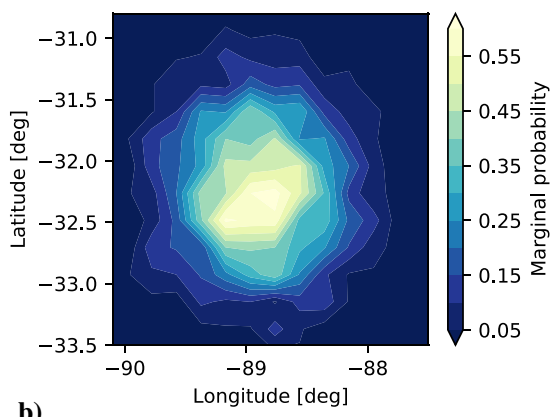

b)

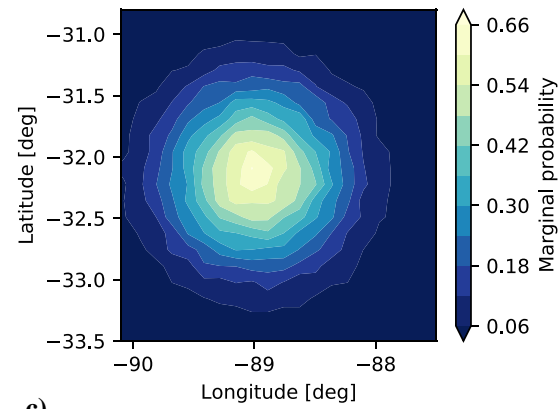

Fig. 20 DB vs MC two-dimensional $\lambda$ - $\varphi$ marginals comparison at landing instant for the Mars reentry test case: a) DB method with 2000 samples and b) MC method with 2000 and c) 50,000 samples.

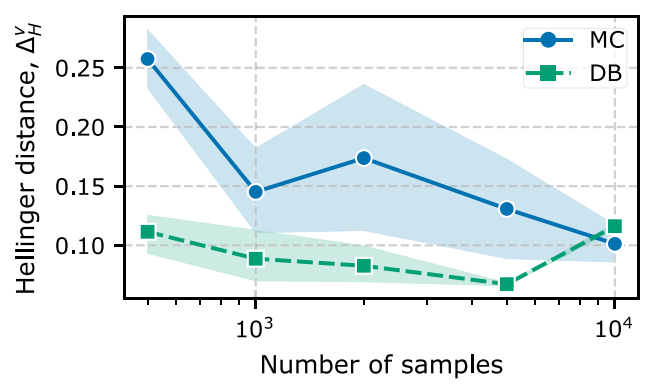

a) Hellinger distance for $v$

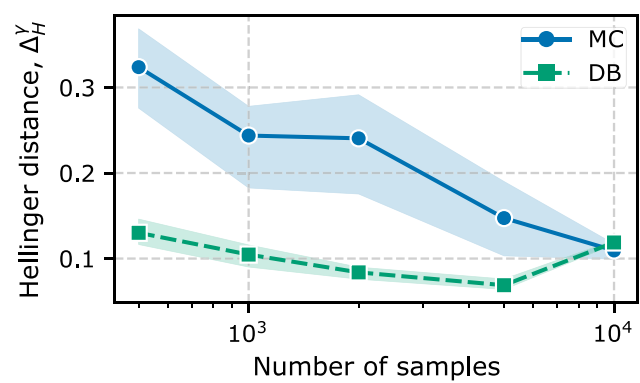

c) Hellinger distance for $\gamma$

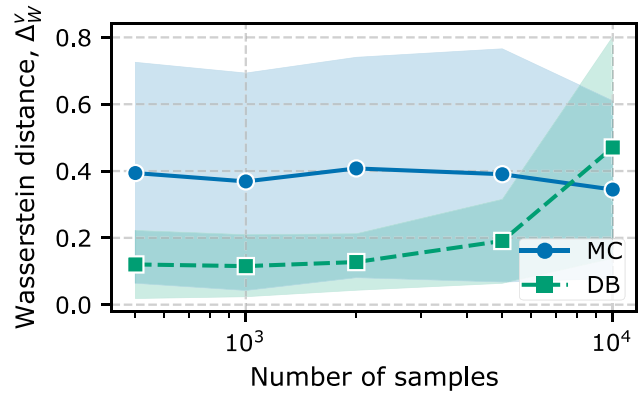

b) Wasserstein distance for $v$

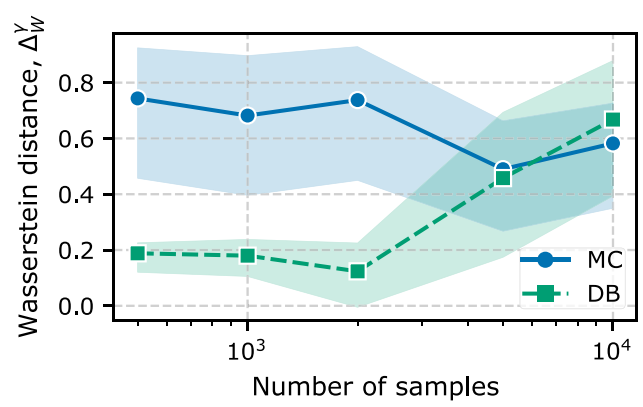

d) Wasserstein distance for $\gamma$

Fig. 21 Comparison of the average Hellinger and Wasserstein distances as a function of the number of samples for the variables $v$ and $\gamma$.

up to 5000 samples, where the DB method performs better than the MC. However, after the 5000 samples, a worsening can be observed, especially in the case of the flight-path angle. We also notice a considerably higher standard deviation for both the DB and MC methods. Contrary to the Hellinger distance, the Wesserstein distance is not a value ranging between 0 and 1 , and its value changes through time alongside the change in magnitude of the probability density. For this reason, in this comparison the value at each snapshot of $\Delta_{W}$ has been normalized so that the average over time could be performed.

The results obtained for the two-dimensional marginals can also be compared using the Hellinger distance and are shown in Table $\underline{6}$. The results shows how the Hellinger distance for the DB method with 1000 samples is comparable with the ones of the MC method with 10,000 samples, confirming the trend observed in the one-dimensional marginals and in previous test cases. A similar trend is also observed in

Table 6 Comparison of Hellinger distance for the $\lambda$ - $\varphi$ marginal at landing between DB and MC methods

\begin{tabular}{lcccc}
\hline \hline \multicolumn{4}{c}{ Number of points } \\
\hline Method & 1000 & 2000 & 5000 & 10,000 \\
DB & 0.12518 & 0.10061 & 0.08712 & 0.0983 \\
MC & 0.27565 & 0.25616 & 0.16781 & 0.11713 \\
\hline \hline
\end{tabular}

the performance of the DB method, with an improvement passing from 1000 to 5000 samples, and then a worsening at 10,000 samples.

Again, Fig. 22 provides a final comparison between the simulation times for the $\overline{\mathrm{DB}}$ and $\mathrm{MC}$ methods. Also in this case, the computational times have been normalized with respect to the 50,000 MC simulation. As expected, the behavior closely matches the one of

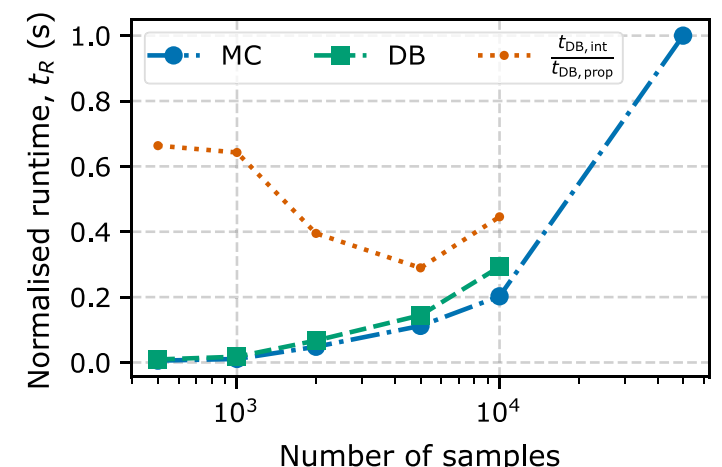

Fig. 22 Run-time comparison between the DB and MC methodology. In orange, the fraction between the interpolation and the propagation times for the DB method. 


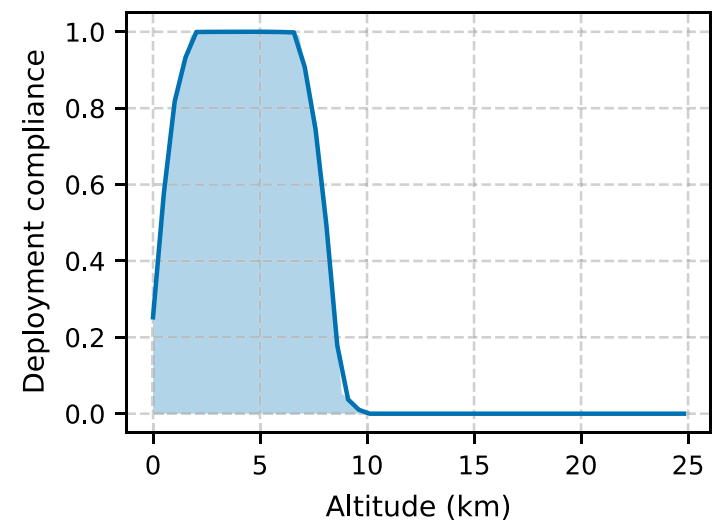

Fig. 23 Parachute deployment compliance probability as a function of the altitude. Only the final part of the trajectory is shown.

Sec. IV.B, while the fraction of computational time devoted to the marginalization compared to the propagation shows a slightly less stable trend: higher fractions of the computational time are occupied by the marginalization procedure for a smaller number of samples.

\section{Parachute Deployment Probability}

Similarly to the test case of Sec. IV.A, in which we analyzed the probability of crossing the thresholds of dynamic pressure and heat rate, in this case, we consider a relevant problem in planetary entry (particularly on Mars) that is the deployment of a parachute to decelerate the probe in the final stage of the reentry. For the parachute deployment, the main variables taken into account are the dynamic pressure and the Mach number. Particularly, the dynamic pressure is limited to $220 \leq \bar{q} \leq 880 \mathrm{~N} / \mathrm{m}^{2}$, and the Mach number is limited to $1.2 \leq M \leq 2.2$ [58]. The dynamic pressure can be computed again using Eq. (27), while the Mach number has the expression

$$
M=\frac{v}{v_{s}}
$$

where $v_{s}$ is the speed of sound in the Martian atmosphere. Similarly to the atmospheric density profile, the speed of sound is considered only a function of the altitude and is obtained from a fitting of MSL data. The expression of the speed of sound as a function of the altitude is [26]

$$
v_{s}(h)=223.8+c_{1} h+c_{2} h^{2}+c_{3} h^{3}
$$

where $c_{1}=-0.2 \times 10^{-3}, c_{2}=-1.588 \times 10^{-8}$, and $c_{3}=1.404 \times 10^{-13}$. Equations (27) and (33) and the deployment boundaries in Mach number and dynamic pressure previously introduced can be combined to obtain the velocity limits within which both the limitations are satisfied. Using these boundaries with the one-dimensional marginals in velocity, we can find the probability of being compliant with the parachute deployment limitations by integrating the velocity marginal within the boundaries. The procedure can be repeated for different snapshots to obtain the evolution of the compliance probability as a function of the altitude. Figure 23 shows the evolution of the compliance probability for the final portion of the trajectory. We can see a full compliance within the altitude range $3-7 \mathrm{~km}$.

Mission planning and design for planetary entries can benefit from such analyses, and they give a robust procedure for selecting the parachute deployment time given the state of the spacecraft under the effect of initial uncertainties.

\section{Conclusions}

This Paper presents a novel methodology to propagate uncertainties using a continuum approach and a procedure to reconstruct these uncertainties and obtain the marginal probabilities of interest. The propagation methodology has been applied to relevant reentry dynamics including both three-state and six-state dynamics and proved capable of including uncertainties in the state variables and in relevant parameters such as the ballistic coefficient and the atmospheric density. The procedure to include this uncertainties, however, requires an extension of the state space of the ODE so that the inclusion of a large number of uncertainty may end up being difficult to achieve at the current stage of development. The reconstruction methodology has been applied to representative test cases to derive the probability marginals for the quantities of interest. The test cases were representative of four-dimensional and six-dimensional state spaces and dynamics with different level of nonlinearity. The DB method showed the capability to obtain the marginal distribution up to a six-dimensional state space that also showed a substantial nonlinear behavior (Figs. 6 and 18). The results were compared with the corresponding Monte Carlo simulations for different number of samples, both in terms of difference between the obtained marginal distributions and simulation times. For the test case of Sec. IV.A, the DB method with 750 samples shows better performance in terms of the Hellinger and Wasserstein distances with respect to the MC method for the first part of the reentry trajectory until about $28 \mathrm{~s}$ into the descent (Fig. 8). Instead, in the last part, the performance of the MC method slightly surpasses the one of the DB method, reaching a comparable performance at the final instant. For the test case of Sec. IV.B, the DB method results in a better performance with respect to the $\overline{\mathrm{MC}}$ simulations with an equivalent number of samples, except for the case with 10,000 samples for the latitude variable (Figs. $16 \mathrm{a}$ and $16 \mathrm{~b}$ ). The DB method also shows a similar or better Wasserstein distance with 1000 samples when compared with the MC method with 5000 and 10,000 samples, while it has a worse performance when 750 samples are considered. The same trend can also be observed for the Hellinger distance for the latitude and velocity variables (Figs. $16 \mathrm{a}$ and $16 \mathrm{c}$ ), but not for the heading angle (Fig. 16e). For the final test case of Sec. IV.C, the DB method showed better or comparable performance than the MC method in approximating the two-dimensional marginals as shown in Table 6 . For the one-dimensional marginals, the DB method showed lower Hellinger distances when compared to the MC method with the same number of samples, except for the case with 10,000 samples (Fig. 21). In addition, the DB method with as low as 750 samples shows, on average, a lower Hellinger distance than the MC method up to 5000 samples (Figs. 21a and 21c). For the same comparison over a one-dimensional marginal, the Wasserstein distance shows a similar behavior, with the DB method performing, on average, better than the MC method for number of samples ranging from 750 to 5000 and instead losing the comparison for 10,000 samples (Figs. 21b and 21d). The computational time of the DB method is always greater than the $\mathrm{MC}$ when the same number of samples is used because the marginalization procedure requires more computational time (Figs. 17 and 22). However, this increase in time can, in some cases, be offset by using a lower number of samples to obtain a comparable level of approximation. The visual inspection of the obtained marginal probabilities showed a trend of the DB method to underpredict the tails of the distributions with respect to the MC method as confirmed by the results of Fig. 11. This trend may be due to the lower number of samples available in these regions of the distributions, thus producing a less accurate reconstruction. In addition, another trend resulting from the presented analyses is the loss of performance of the DB method when a larger number of samples is used (i.e., 10,000 samples in this Paper). This is a limitation of the introduced marginalization technique, which requires the definition of bins; if these bins are too small, the volume approximated by the triangulation may be inaccurate. However, a finer binning is usually required when the number of samples points increases (especially in higher dimensions) to manage the memory usage of the triangulation algorithm. This can limit the applicability of the reconstruction methodology to high-dimensional spaces. Still, the methodology has good performance when lower number of samples is used, and this is also where it is most applicable and appealing.

\section{Acknowledgments}

This project has received funding from the European Research Council under the European Union's Horizon 2020 research and innovation programme (grant agreement 679086-COMPASS). The data 
generated for this study can be found in the repository at the link www. compass.polimi.it/publications.

\section{References}

[1] Hoelscher, B., "Orion Entry, Descent and Landing Simulation," AIAA Guidance, Navigation and Control Conference and Exhibit, AIAA Paper 2007-6428, 2007.

https://doi.org/10.2514/6.2007-6428

[2] Rea, J., and Putnam, Z., "A Comparison of Two Orion Skip Entry Guidance Algorithms," AIAA Guidance, Navigation and Control Conference and Exhibit, AIAA Paper 2007-6424, 2007.

[3] Putnam, Z., Bairstow, S., Braun, R., and Barton, G., "Improving Lunar Return Entry Range Capability Using Enhanced Skip Trajectory Guidance," Journal of Spacecraft and Rockets, Vol. 45, No. 2, 2008, pp. 309-315. https://doi.org/10.2514/1.27616

[4] Brunner, C. W., and Lu, P., "Skip Entry Trajectory Planning and Guidance," Journal of Guidance, Control, and Dynamics, Vol. 31, No. 5, 2008, pp. 1210-1219.

[5] Putnam, Z. R., Grant, M. J., Kelly, J. R., Braun, R. D., and Krevor, Z. C., "Feasibility of Guided Entry for a Crewed Lifting Body Without Angleof-Attack Control," Journal of Guidance, Control, and Dynamics, Vol. 37, No. 3, 2014, pp. 729-740. https://doi.org/10.2514/1.62214

[6] Spencer, D. A., and Braun, R. D., "Mars Pathfinder Atmospheric EntryTrajectory Design and Dispersion Analysis," Journal of Spacecraft and Rockets, Vol. 33, No. 5, 1996, pp. 670-676. https://doi.org/10.2514/3.26819

[7] Striepe, S. A., Way, D., Dwyer, A., and Balaram, J., "Mars Science Laboratory Simulations for Entry, Descent, and Landing," Journal of Spacecraft and Rockets, Vol. 43, No. 2, 2006, pp. 311-323. https://doi.org/10.2514/1.19649

[8] Nelessen, A., Sackier, C., Clark, I., Brugarolas, P., Villar, G., Chen, A., Stehura, A., Otero, R., Stilley, E., Way, D., Edquist, K., Mohan, S., Giovingo, C., and Lefland, M., "Mars 2020 Entry, Descent, and Landing System Overview," 2019 IEEE Aerospace Conference, IEEE, New York, 2019, pp. 1-20.

https://doi.org/10.1109/AERO.2019.8742167

[9] Pardini, C., and Anselmo, L., "Re-Entry Predictions for Uncontrolled Satellites: Results and Challenges," 6th International Association for Space Safety Conference, ESA Communications, European Space Agency, ESTEC, ESA SP-715, Noordwijk, The Netherlands, Sept. 2013.

[10] O'Connor, B., Handbook for Limiting Orbital Debris. NASA Handbook 8719.14, NASA, 2008, pp. 153-174.

[11] Dobarco-Otero, J., Smith, R., Bledsoe, K., Delaune, R., Rochelle, W., and Johnson, N., "The Object Reentry Survival Analysis Tool (ORSAT)Version 6.0 and Its Application to Spacecraft Entry," Proceedings of the 56th Congress of the International Astronautical Federation, International Academy of Astronautics and International Inst. of Space Law, IAC-05-B6, Vol. 3, Paris, France, 2005, pp. 17-21.

[12] Koppenwallner, G., Fritsche, B., Lips, T., and Klinkrad, H., "Scarab-a Multi-Disciplinary Code for Destruction Analysis of Space-Craft during Re-Entry," Fifth European Symposium on Aerothermodynamics for Space Vehicles, Vol. 563, European Space Agency, Noordwijk, The Netherlands, 2005, pp. 281-287.

[13] Gelhaus, J., Kebschull, C., Braun, V., Sanchez-Ortiz, N., Parilla Endrino, E., de Oliveira, J. C., and Dominguez-Gonzalez, R., "Upgrade of ESA's Space Debris Mitigation Analysis Tool Suite," ESA TR 4000104977/11/D/SR, 2014.

[14] Trisolini, M., Lewis, H. G., and Colombo, C., "Demisability and Survivability Sensitivity to Design-for-Demise Techniques," Acta Astronautica, Vol. 145, April 2018, pp. 357-384. https://doi.org/10.1016/j.actaastro.2018.01.050

[15] Niederreiter, H., Random Number Generation and Quasi-Monte Carlo Methods, Vol. 63, Soc. for Industrial and Applied Mathematics, Philadelphia, PA, 1992, pp. 1-12.

[16] Luo, Y.-Z., and Yang, Z., "A Review of Uncertainty Propagation in Orbital Mechanics," Progress in Aerospace Sciences, Vol. 89, Feb. 2017, pp. 23-39. https://doi.org/10.1016/j.paerosci.2016.12.002

[17] Cameron, J. M., Jain, A., Burkhart, P. D., Bailey, E. S., Balaram, B., Bonfiglio, E., Ivanov, M., Benito, J., Sklyanskiy, E., and Strauss, W., "DSENDS: Multi-Mission Flight Dynamics Simulator for NASA Missions," AIAA SPACE 2016, AIAA Paper 2016-5421, 2016. https://doi.org/10.2514/6.2016-5421

[18] Hanson, J. M., and Jones, R. E., "Test Results for Entry Guidance Methods for Space Vehicles," Journal of Guidance, Control, and
Dynamics, Vol. 27, No. 6, 2004, pp. 960-966. https://doi.org/10.2514/1.10886

[19] Lu, P., "Predictor-Corrector Entry Guidance for Low-Lifting Vehicles," Journal of Guidance, Control, and Dynamics, Vol. 31, No. 4, 2008, pp. 1067-1075. https://doi.org/10.2514/1.32055

[20] Lu, P., Brunner, C. W., Stachowiak, S. J., Mendeck, G. F., Tigges, M. A., and Cerimele, C. J., "Verification of a Fully Numerical Entry Guidance Algorithm," Journal of Guidance, Control, and Dynamics, Vol. 40, No. 2, 2017, pp. 230-247. https://doi.org/10.2514/1.G000327

[21] Mehta, P. M., Kubicek, M., Minisci, E., and Vasile, M., "Sensitivity Analysis and Probabilistic Re-Entry Modeling for Debris Using High Dimensional Model Representation Based Uncertainty Treatment," Advances in Space Research, Vol. 59, No. 1, 2017, pp. 193-211. https://doi.org/10.1016/j.asr.2016.08.032

[22] Sanson, F. J., Bertorello, C., Bouilly, J.-M., and Congedo, P., "Space Debris Reentry Prediction and Ground Risk Estimation Using a Probabilistic Breakup Model," AIAA Scitech 2019 Forum, AIAA Paper 2019-2234, 2019. https://doi.org/10.2514/6.2019-2234

[23] Dell'Elce, L., and Kerschen, G., "Probabilistic Assessment of Lifetime of Low-Earth-Orbit Spacecraft: Uncertainty Propagation and Sensitivity Analysis," Journal of Guidance, Control, and Dynamics, Vol. 38, No. 5, 2015, pp. 886-899. https://doi.org/10.2514/1.G000149

[24] Prabhakar, A., Fisher, J., and Bhattacharya, R., "Polynomial ChaosBased Analysis of Probabilistic Uncertainty in Hypersonic Flight Dynamics," Journal of Guidance, Control, and Dynamics, Vol. 33, No. 1, 2010, pp. 222-234. https://doi.org/10.2514/1.41551

[25] Jones, B. A., and Doostan, A., "Satellite Collision Probability Estimation Using Polynomial Chaos Expansions," Advances in Space Research, Vol. 52, No. 11, 2013, pp. 1860-1875. https://doi.org/10.1016/j.asr.2013.08.027

[26] Lunghi, P., Armellin, R., Di Lizia, P., Mease, K. D., and Lavagna, M. R., "Atmospheric Entry Guidance Based on Differential Algebra for High Elevation Mars Landing," 2018 Space Flight Mechanics Meeting, AIAA Paper 2018-1458, 2018. https://doi.org/10.2514/6.2018-1458

[27] Chandrasekhar, S. S., Principles of Stellar Dynamics, Dover, New York, 1943, Chap. 3.

[28] Gor'kavyi, N. N., Ozernoy, L. M., and Mather, J. C., "A New Approach to Dynamical Evolution of Interplanetary Dust," Astrophysical Journal, Vol. 474, No. 1, 1997, pp. 496-502. https://doi.org/10.1086/303440

[29] McInnes, C. R., "Autonomous Ring Formation for a Planar Constellation of Satellites," Journal of Guidance, Control, and Dynamics, Vol. 18, No. 5, 1995, pp. 1215-1217. https://doi.org/10.2514/3.21531

[30] Letizia, F., "Extension of the Density Approach for Debris Cloud Propagation," Journal of Guidance, Control, and Dynamics, Vol. 41, No. 12, 2018, pp. 2651-2657. https://doi.org/10.2514/1.G003675

[31] Frey, S., Colombo, C., and Lemmens, S., "Interpolation and Integration of Phase Space Density for Estimation of Fragmentation Cloud Distribution," Proceedings of the 29th AAS/AIAA Space Flight Mechanics Meeting, Vol. 168, 2019, pp. 2229-2240.

[32] Halder, A., and Bhattacharya, R., "Dispersion Analysis in Hypersonic Flight During Planetary Entry Using Stochastic Liouville Equation," Journal of Guidance, Control, and Dynamics, Vol. 34, No. 2, 2011, pp. 459-474. https://doi.org/10.2514/1.51196

[33] Trisolini, M., and Colombo, C., "A Density-Based Approach to the Propagation of Re-Entry Uncertainties," Advances in the Astronautical Sciences, Vol. 168, 2019, pp. 2241-2253.

[34] Trisolini, M., and Colombo, C., "Modeling Re-Entry Break-Up Uncertainties with Continuity Equation and Gaussian Mixture Models Interpolation," Proceedings of the 2020 AAS/AIAA Astrodynamics Specialist Conference-Virtual Lake Tahoe, AAS Paper 20-636, Lake Tahoe, USA, Aug. 2020.

[35] Limonta, S., Trisolini, M., Frey, S., and Colombo, C., "Modelling the Break-Up and Re-Entry Propagation of Meteorites Through a Continuum Approach," Proceedings of the 71st International Astronautical Congress (IAC)-The Cyber Space Edition, Paper IAC-20-C1.6.10, Oct. 2020

[36] Hoogendoorn, R., Mooij, E., and Geul, J., "Uncertainty Propagation for Statistical Impact Prediction of Space Debris," Advances in Space Research, Vol. 61, No. 1, 2018, pp. 167-181. https://doi.org/10.1016/j.asr.2017.10.009 
[37] Stoer, J., and Bulirsch, R., "Interpolation," Introduction to Numerical Analysis, Springer-Verlag, New York, 2002, pp. 37-144. https://doi.org/10.1007/978-0-387-21738-3_2

[38] Edelsbrunner, H., and Mücke, E. P., "Three-Dimensional Alpha Shapes," ACM Transactions on Graphics, Vol. 13, No. 1, 1994, pp. 43-72. https://doi.org/10.1145/174462.156635

[39] Kraaijpoel, D., and Van Leeuwen, T., "Raising the Order of Multivariate Approximation Schemes Using Supplementary Derivative Data," Procedia Computer Science, Vol. 1, Elsevier, Amsterdam, The Netherlands, 2010, pp. 307-316.

https://doi.org/10.1016/j.procs.2010.04.034

[40] Awanou, G., Lai, M.-J., and Wenston, P., "The Multivariate Spline Method for Scattered Data Fitting and Numerical Solutions of Partial Differential Equations," Wavelets and Splines: Athens, Nashboro Press, Brentwood, TN, 2005, pp. 24-74.

[41] Alfeld, P., "Scattered Data Interpolation in Three or More Variables," Mathematical Methods in Computer Aided Geometric Design, Elsevier, Amsterdam, The Netherlands, 1989, pp. 1-33. https://doi.org/10.1016/B978-0-12-460515-2.50005-6

[42] Barthelmann, V., Novak, E., and Ritter, K., "High Dimensional Polynomial Interpolation on Sparse Grids," Advances in Computational Mathematics, Vol. 12, No. 4, 2000, pp. 273-288. https://doi.org/10.1023/A:1018977404843

[43] Buhmann, M. D., Radial Basis Functions: Theory and Implementations, Vol. 12, Cambridge Univ. Press, Cambridge, England, U.K., 2003, pp. 99-146.

[44] Preparata, F. P., and Shamos, M. I., Computational Geometry, SpringerVerlag, New York, 1985, pp. 191-218. https://doi.org/10.1007/978-1-4612-1098-6

[45] Virtanen, P., Gommers, R., Oliphant, T. E., Haberland, M., Reddy, T., Cournapeau, D., Burovski, E., Peterson, P., Weckesser, W., Bright, J., van der Walt, S. J., Brett, M., Wilson, J., Millman, K. J., Mayorov, N., Nelson, A. R. J., Jones, E., Kern, R., Larson, E., Carey, C. J., Polat, I., Feng, Y., Moore, E. W., VanderPlas, J., Laxalde, D., Perktold, J., Cimrman, R. Henriksen, I., Quintero, E. A., Harris, C. R., Archibald, A. M., Ribeiro, A. H., Pedregosa, F., and van Mulbregt, P., and SciPy 1.0 Contributors, "SciPy 1.0: Fundamental Algorithms for Scientific Computing in Python," Nature Methods, Vol. 17, Feb. 2020, pp. 261-272. https://doi.org/10.1038/s41592-019-0686-2

[46] Coxeter, H. S. M., Regular Polytopes, Courier Dover, New York, 1973, Chap. VII.

[47] Storn, R., and Price, K., "Differential Evolution-A Simple and Efficient Heuristic for Global Optimization over Continuous Spaces," Journal of Global Optimization, Vol. 11, No. 4, 1997, pp. 341-359. https://doi.org/10.1023/A:1008202821328
[48] Schmidt, M., Safarani, S., Gastinger, J., Jacobs, T., Nicolas, S., and Schülke, A., "On the Performance of Differential Evolution for Hyperparameter Tuning," 2019 International Joint Conference on Neural Networks (IJCNN), IEEE, New York, 2019, pp. 1-8. https://doi.org/10.1109/IJCNN.2019.8851978

[49] Mezura-Montes, E., Reyes-Sierra, M., and Coello, C. A. C., "MultiObjective Optimization Using Differential Evolution: A Survey of the State-of-the-Art," Advances in Differential Evolution, Springer-Verlag, Berlin, 2008, pp. 173-196.

https://doi.org/10.1007/978-3-540-68830-3_7

[50] Teichmann, M., and Capps, M., "Surface Reconstruction with Anisotropic Density-Scaled Alpha Shapes," Proceedings Visualization'98 (Cat. No. 98CB36276), IEEE, New York, 1998, pp. 67-72. https://doi.org/10.1109/VISUAL.1998.745286

[51] Putnam, Z. R., and Braun, R. D., "Extension and Enhancement of the Allen-Eggers Analytical Ballistic Entry Trajectory Solution," Journal of Guidance, Control, and Dynamics, Vol. 38, No. 3, 2015, pp. 414-430. https://doi.org/10.2514/1.G000846

[52] Kemp, N. H., and Riddell, F., "Heat Transfer to Satellite Vehicles ReEntering the Atmosphere," Journal of Jet Propulsion, Vol. 27, No. 2, 1957, pp. 132-137. https://doi.org/10.2514/8.12603

[53] Beck, J., Merrifield, J., Holbrough, I., Markelov, G., and Molina, R., "Application of the SAM Destructive Re-Entry Code to the Spacecraft Demise Integration Test Cases," 8th European Symposium on Aerothermodynamics of Space Vehicles, European Space Agency, Noordwijk, The Netherlands, 2015.

[54] Trisolini, M., Lewis, H. G., and Colombo, C., "Spacecraft Design Optimisation for Demise and Survivability," Aerospace Science and Technology, Vol. 77, June 2018, pp. 638-657. https://doi.org/10.1016/j.ast.2018.04.006

[55] Lu, P., "Entry Guidance: A Unified Method," Journal of Guidance, Control, and Dynamics, Vol. 37, No. 3, 2014, pp. 713-728. https://doi.org/10.2514/1.62605

[56] Tewari, A., Atmospheric and Space Flight Dynamics: Modeling and Simulation with MATLAB® and Simulink ${ }^{\circledR}$, Birkhäuser, Basel, Switzerland, 2007, pp. 45-58.

[57] National Oceanic and Atmopsheric Administration, National Aeronautics and Space Administration, United States Air Force, "U.S. Standard Atmosphere 1976," U.S. Government Printing Office, Rept. NOAA-S/T76-1562, NASA-TM-X-74335, Washington, D.C., Oct. 1976, pp. 1-227.

[58] Kluever, C., "Entry Guidance Performance for Mars Precision Landing," Journal of Guidance, Control, and Dynamics, Vol. 31, No. 6, 2008, pp. 1537-1544.

https://doi.org/10.2514/1.36950 